\title{
A framework for space-efficient variable-order Markov models
}

\author{
Fabio Cunial* $\quad$ Jarno Alanko ${ }^{\dagger} \quad$ Djamal Belazzougui ${ }^{\ddagger}$
}

\section{Abstract}

Motivation: Markov models with contexts of variable length are widely used in bioinformatics for representing sets of sequences with similar biological properties. When models contain many long contexts, existing implementations are either unable to handle genome-scale training datasets within typical memory budgets, or they are optimized for specific model variants and are thus inflexible.

Results: We provide practical, versatile representations of variable-order Markov models and of interpolated Markov models, that support a large number of context-selection criteria, scoring functions, probability smoothing methods, and interpolations, and that take up to 4 times less space than previous implementations based on the suffix array, regardless of the number and length of contexts, and up to 10 times less space than previous trie-based representations, or more, while matching the size of related, state-of-the-art data structures from Natural Language Processing. We describe how to further compress our indexes to a quantity related to the redundancy of the training data, saving up to $90 \%$ of their space on repetitive datasets, and making them become up to 60 times smaller than previous implementations

\footnotetext{
* Max Planck Institute for Molecular Cell Biology and Genetics (MPI-CBG), and Center for Systems Biology Dresden (CSBD), Dresden, 01307, Germany. cunial@mpi-cbg.de

${ }^{\dagger}$ Department of Computer Science, University of Helsinki, Helsinki, 00014, Finland.

${ }^{\ddagger}$ CAPA, DTISI, Centre de Récherche sur l'Information Scientifique et Technique, Algiers, Algeria.
}

based on the suffix array. Finally, we show how to exploit constraints on the length and frequency of contexts to further shrink our compressed indexes to half of their size or more, achieving data structures that are 100 times smaller than previous implementations based on the suffix array, or more. This allows variable-order Markov models to be trained on bigger datasets and with longer contexts on the same hardware, thus possibly enabling new applications.

Availability and implementation: https://github.com/jnalanko/VOMM

\section{Introduction}

Building statistical models for large sets of sequences that share biological properties is a fundamental problem in bioinformatics, with connections to coding, compression, and machine learning. In many biological sequences, the empirical probability distribution of the next character does not change significantly if one takes into account a subsequence of the recent history (called the context of the character) that is longer than a fixed threshold, thus Markov models in which all states are strings of uniform length $k$, called the order of the model, are routinely used. Choosing the order of a Markov model asks one to balance between conflicting issues. Longer contexts imply estimating exponentially more parameters, which might not be feasible with the available data and which might hurt generalization. However, while most long contexts might be too infrequent to yield accurate 
probability estimates, a few of them might still occur frequently enough to be used as predictors in practice. Shorter contexts, on the other hand, yield models of tractable size, but they might lead to inaccurate predictions, since they are unable to capture long-range dependencies. Variable-order Markov models (VOMMs, [42]) aim at solving such issues by allowing the length of the context used to predict the next character to depend on the recently observed characters themselves. This dependency, called local order, has indeed been observed in a number of natural sources [8]. A VOMM can thus be seen as a compression of a fixed-order Markov model of large order, in which all states that yield similar character distributions are collapsed into a single state, whose sequence is decided by a number of statistical criteria.

Variable-order Markov models and their variants, like interpolated Markov models (IMMs, see e.g. [46]), are by now a staple of bioinformatics, and have been successfully applied to detect domains in protein sequences [4], to segment protein sequences into domains $[50,8,6]$, to assign domains and proteins to families [4], to separate coding from non-coding DNA regions [45], to detect horizontal gene transfer [21], to identify genes in newly sequenced microbial genomes after being trained on known open reading frames [46, 24, 25], and to detect eukaryotic promoters [39]. VOMMs and their variants have been applied to metagenomic samples as well. For example, VOMMs have been used to separate the reads of a eukaryotic host from those of an intracellular prokaryotic parasite [24]; to model known genomes in order to estimate, given a metagenomic sample, the genome or taxon a read was sampled from [12]; to define compositional distances between metatranscriptomic samples [32]; and to model the clusters produced by reference-free binning of metagenomic reads $[28,56,58]$.

As mentioned, a key reason for the introduction of VOMMs is the implicit compression they perform over a Markov model of fixed, large order [42, 62]. Space-efficient implementations of VOMMs and of their variants are becoming increasingly relevant in the post-genome era, in which one wishes to train them on long genomes, on large metagenomic samples, or on massive collections of genomes of similar species or of proteins with the same function or structure $[6,7,8,28]$. When the criteria for deciding whether a subsequence of the training data should be used as a context are so stringent that just a small set of short subsequences is chosen, the space taken by a VOMM is not a practical issue, and a simple pointer-based trie implementation like e.g. the one described by [5] suffices. In this paper we focus on VOMMs in which the total length of all contexts is large enough to make such a simple approach impractical. This is a concrete scenario, especially in large datasets, since e.g. increasing the maximum length of a context does not decrease classification performance in practice [4], and in real use cases one wishes to experiment with multiple selection criteria without worrying about blowups in space or training time.

The first training algorithms required $O\left(k|T|^{2}\right)$ time for building a VOMM from a sequence $T$, where $k$ is an upper bound on context length, and $O\left(|S|^{2}\right)$ time to compute the probability of a query sequence $S$ according to the model [45]. Later, [1] described a general-purpose data structure, based on the suffix tree of $T$, that can be built in $O(|T|)$ time for alphabets of constant size $\sigma$ and for a large number of context selection criteria, and that allows one to score a query $S$ in $O(|S|)$ time for constant alphabets. Such data structure was the first to take $O(|T| \log |T|)$ bits of space, regardless of the number and length of contexts, and it was later implemented using lazy suffix trees or enhanced suffix arrays $[48,49]$. However, such implementations are not available any more [47]. [33] designed and implemented another data structure, based on the suffix array and the inverse suffix array of $T$, that takes again $O(|T| \log |T|)$ bits of space, but that allows scoring a query using as context just the longest match with the training data at each position. Meanwhile, IMMs with ad hoc emission probability formulas were being deployed in natural language processing (NLP), and compact indexes for computing a 
specific family of such probabilities have been developed and implemented at scale by [52, 53]. Such data structures rely on compressed suffix trees, and thus need just $O(|T| \log \sigma)$ bits of space. However, they do not implement generalpurpose VOMMs, and they are optimized to answer queries on the probability of observing a character after an arbitrarily large number $\ell$ of other characters specified in the query, rather than to score an entire sequence $S$, thus they take $O(\ell|S|)$ time to compute such global score in the worst case (although practical implementations try to reuse counts across adjacent sliding windows). More recently, [10] described a general-purpose data structure that takes just $3|T| \log \sigma+o(|T| \log \sigma)$ bits of space to implement a VOMM or an IMM, regardless of the number and length of contexts and for a large set of context selection criteria, and that can be trained in $O(|T|)$ time using $O(|T| \log \sigma)$ bits of working space. In theory the space taken by such data structure is similar to the space taken by the data structures by $[52,53]$, while at the same time supporting a variety of context selection and probability smoothing criteria, and allowing one to score a query $S$ in $O(|S|)$ time using $2|S|+o(|S|)$ bits of space.

This paper is concerned with building practical, general-purpose representations of VOMMs and IMMs that are as small as possible. Optimizing query time and the construction of such representations is outside the scope of the paper. Our first contribution is an implementation of the theory described in [10]: we provide a general-purpose framework of practical data structures and algorithms that allows bioinformaticians to implement a large number of VOMMs and IMMs in small space, including many context-selection criteria, scoring functions, probability smoothing methods, and interpolations, without affecting the accuracy of the models and scaling to large datasets. No such framework currently exists, either because available implementations cannot handle large datasets within typical memory budgets, or because they support just few specific Markov models. We implement a representative subset of all the variants that our framework supports, achieving up to approximately 4 times less space than [33] and 10 times less space (or even less, depending on context selection criterion) than [5], while matching the space of the NLP data structures in $[52,53]$. Since VOMMs are generative statistical models, their training datasets consist typically of positive examples of a class, and are thus likely to be repetitive. The second contribution of this paper is an extension of the approach in [10] which, for the first time, reduces the size of a VOMM to a quantity related to the redundancy of the training data. In repetitive datasets, this allows saving up to approximately $90 \%$ of the space of our index, without any effect on accuracy, making our data structures become up to approximately 60 times smaller than [33]. Finally, we describe a way of shrinking our data structures even further when the user sets an upper bound on context length, or a lower bound on context frequency, which are common in applications (see e.g. $[18,45,46]$ ). This can shrink our already compressed index to half of its size and beyond, depending on the dataset, yielding a data structure that is up 100 times or more smaller than [33], again without affecting accuracy. Such space reductions apply to a large number of VOMM and IMM variants, albeit not to all of them, and, as expected, they come at the cost of increased scoring time: even the basic version of our index is between 35 and 60 times slower than [5], and between 3 and 12 times slower than [33].

\section{Preliminaries and notation}

\subsection{Strings and string indexes}

Let $\Sigma=[1 . . \sigma]$ be an integer alphabet, let $\#=0$ be a separator not in $\Sigma$, and let $S$ and $T$ be strings in $[1 . . \sigma]^{*}$. The matching statistics array $\mathrm{MS}_{S, T}$ is an array of length $|S|$ such that $\mathrm{MS}_{S, T}[i]$ is the largest $\ell$ such that $S[i-\ell+1 . . i]$ occurs in $T$. We denote by $\bar{W}$ the reverse of a string $W$, i.e. string $W$ read from right to left. We denote by $f_{T}(W)$ the number of (possibly overlapping) occurrences of a string $W$ in $T$, we denote by $\Sigma_{T}^{r}(W)$ the set of distinct characters that occur to the right of $W$ in $T$, and we de- 
note by $\Sigma_{T}^{r}(W, t)$ (respectively, by $\left.\Sigma_{T}^{r}(W, \geq t)\right)$ the set of distinct characters that occur exactly (respectively, at least) $t$ times to the right of $W$ in $T$. The characters in $\Sigma_{T}^{r}(W)$ are called right-extensions of $W$, and $\Sigma_{T}^{\ell}(W), \Sigma_{T}^{\ell}(W, t)$ and $\Sigma_{T}^{\ell}(W \geq t)$ are defined symmetrically for the left side of $W$. We use small sigma as a shorthand for the cardinality of every such set, e.g. $\sigma_{T}^{r}(W)=\left|\Sigma_{T}^{r}(W)\right|$. We call empirical emission probability of a character $a$ from a substring $W$ of $T$ the quantity $P_{T}(a \mid W)=f_{T}(W a) / f_{T}(W)$, although a number of other estimates have been proposed, for example the modified Laplace rule $P(a \mid W)=(f(W a)+0.5) /(f(W)+\sigma / 2)$ and $P(a \mid W)=f(W a) /\left(f(W)+\sigma^{r}(W)\right)$ (see e.g. $[1,3,62,64,40,8,4,2,18])$. We call empirical probability of a string $W$ in $T$ the quantity $P_{T}(W)=f_{T}(W) /(|T|-|W|+1)$. A repeat $W$ of $T$ is a substring of $T$ that satisfies $f(W)>1 . W$ is right-maximal (respectively, left-maximal) iff $\sigma_{T \#}^{r}(W)>1$ (respectively, iff $\sigma_{T}^{\ell}(W)>1$ ). We call right-deterministic (respectively, left-deterministic) a string that is not right-maximal (respectively, left-maximal). It is well known that $T$ can have at most $|T|-1$ rightmaximal repeats and at most $|T|-1$ left-maximal repeats. A maximal repeat is a repeat that is both left- and right-maximal.

We assume the reader to be familiar with standard notions from text indexes, including the suffix tree $\mathrm{ST}_{T}$ and the suffix-link tree $\mathrm{SLT}_{T}$ of a string $T$, Weiner links, the Burrows-Wheeler transform $\mathrm{BWT}_{T}$, the lexicographic interval of a right-maximal repeat in the BWT, backward steps, rank and select queries, wavelet trees, and compact representations of tree topologies. See Section 9 in the supplement for more details and for pointers to the literature. In the rest of the paper, we omit subscripts whenever they are clear from the context.

\subsection{Variable-order Markov models}

We assume the reader to be familiar with fixedorder Markov models, which we do not define or further describe here. Given a Markov model of fixed order $k$ on alphabet $[1 . . \sigma]$, let $\mathbb{P}(a \mid X)$ be the emission probability of state $X$, i.e. the proba- bility of observing character $a$ after the length- $k$ context $X$. Given a string $W$ of length $h<k$, it might happen that $\mathbb{P}(a \mid U W)=\mathbb{P}(a \mid V W)$ for all strings $U$ and $V$ of length $k-h$ and for all characters $a$ : in other words, adding up to $k-h$ characters before $W$ does not alter the probability of seeing any character after $W$. This is called variable-order Markov model (VOMM), and it can be represented more compactly by storing just $W$ (rather than all $k$-mers with $W$ as a suffix) and the emission probabilities of $W$ [42]. In what follows we assume that a Markov model is built from a training string $T$. The set of contexts is typically stored in a trie $\mathcal{T}$, called context tree or probabilistic suffix tree (see e.g. [23, 7] and references therein), where contexts are inserted from right to left, and in which every node that corresponds to a context is marked and stores emission probabilities [45]. We denote by $\mathcal{C}$ the set of nodes of $\mathcal{T}$ that are marked as contexts (abusing notation, we use symbol $\mathcal{C}$ to denote also the set of contexts).

In the following sections we survey a number of criteria used to assign a probability to a query, and to select contexts from substrings of the training data. As we will see in Section 4, most such criteria can be implemented with a small setup of space-efficient data structures.

\subsubsection{Scoring a query}

The probability of a query string $S$ according to the VOMM is computed character by character, as follows: at every position $j$ of $S$, the probability of character $S[j]=b$ is decided by finding a context $w \in \mathcal{C}$ based on $S[1 . . j-1]$, and by accessing the empirical probability $P(b \mid W)$ stored at $w$ $[43,42]$. The function that returns the context to be used at position $j$ is called structure function [43], and it typically returns the longest context that is a suffix of $S[1 . . j-1]$. The probability of $S$ is then the product of the probability of every character of $S$ (in the rest of the paper we focus on computing log-probabilities, thus all products become additions). Some variants multiply the probability of $S$ by the average value of $\sigma^{r}(W)$ over all contexts $W$ used for prediction [37].

In most applications, when $W$ does not emit 
$b$ in the model, the probability of character $S[j]$ should still be set to a nonzero value: deciding such nonzero value is called the zero-frequency or the sparse data problem $[18,64,4]$, and taking this into account to adapt the probability of characters that are emitted by the model is called smoothing. Such nonzero value can be a user-defined constant, a function of $f(b), f(W)$, $\sigma, \sigma^{r}(W)$ or $\sigma^{r}(W, 1)$, like the Good-Turing estimator (see e.g. $[42,4,18,17])$, or it can be computed recursively. For example, in the conceptually similar PPM compression algorithm [18], one assigns to $S[j]$ a probability $\tilde{P}(b \mid W)$ defined as follows [3]: $\tilde{P}(b \mid W)=P(b \mid W) \cdot(1-$ $\hat{P}(W))$ if $W \in \mathcal{C}$ and $P(b \mid W)>0, \tilde{P}(b \mid W)=$ $\hat{P}(W) \cdot \tilde{P}(b \mid W[2 . .|W|])$ otherwise, where $\hat{P}(W)$, called escape or backoff probability, is an estimate of the probability of observing a character that does not follow $W$ in the model, for example the Good-Turing estimator mentioned above (see e.g. [3, 18, 64] and references therein). In some approaches, recursion stops when the suffix reaches a user-specified minimum length $[39,46,13]$ or frequency [46].

A similar approach can be applied even when $W$ emits $b$, to compute a mixture of probability estimates (see e.g. [16, 46]), possibly with weights that depend on the length of the contexts [23]: this is also called blending [35]. An interpolated Markov model (IMM) is a fixedorder Markov model in which emission probabilities are a weighted mixture of lower-order contexts [46, 39], for example $\tilde{P}(b \mid W)=\lambda(W)$. $P(b \mid W)+(1-\lambda(W)) \cdot \tilde{P}(b \mid W[2 . .|W|])$ if $|W|>$ 0 , and otherwise it is a function of quantities like $f(b),|T|, \sigma$ and $\sigma^{r}(W)$ (see e.g. [16]). Weights $\lambda(W)$ can represent the confidence in the accuracy of an emission probability estimate (longer contexts yield stronger predictions, but shorter contexts have more accurate statistics), or the prior that correlation decreases with distance $[23,22,17]$. Weights can be a fixed vector of constants (which might sum to one), or a given function of the frequency, length, and number of right-extensions of every suffix (see e.g. $[39,60,46,44,16])$. More complex variants of recursive emission probability functions have been designed in natural language model- ing and information retrieval, e.g. Kneser-Ney smoothings (see e.g. $[52,53,51,30]$ and references therein). Non-recursive ways of interpolating the probabilities of different contexts have also been proposed. For example, one can set $\tilde{P}(a \mid W)=(f(W a)-x) / f(W)+\alpha(W) \beta(W, a)$ if $f(W a)>0$, and $\tilde{P}(a \mid W)=\alpha(W) \beta(W, a)$ otherwise, where $x$ is a parameter such that $x<f(W c)$ for all $c \in \Sigma^{r}(W), \beta(W, a)=$ $\sigma^{\ell}(W[2 . .|W|] a) / f(W[2 . .|W|])$ is a back-off function, and $\alpha(W)=\sigma^{r}(W) \cdot x / f(W)[29]$. Yet other variants take the maximum emission probability over all possible contexts, rather than blending context probabilities together [37].

\subsubsection{Selecting contexts}

A number of algorithms for learning context trees optimally in a statistical sense have been described (see e.g. [22, 63, 45, 27, 15], including methods that exploit connections with the suffix tree and BWT [2, 35]. Here we focus on criteria that select a substring $W$ of the training data as a context based only on properties of $W$. Combinatorial approaches mark all substrings as contexts [33] and select the longest context at each position of the query, or alternatively mark all right-deterministic substrings as contexts [59, 19], and start prediction from the shortest context at each position of the query (if any). Statistical approaches mark as contexts all the frequent substrings $a W$ (where $a \in[1 . . \sigma]$ and $\left.W \in[1 . . \sigma]^{*}\right)$ having at least one character $b$ with high emission probability from $a W$, and such that the emission probability of $b$ from $a W$ is significantly different from the emission probability of $b$ from $W$ (see e.g. [7] and references therein). Specifically, for user-defined thresholds $\tau_{1}>0, \tau_{2}>0, \tau_{3}<1$ and $\tau_{4}>1$, they require $P(a W) \geq \tau_{1}, P(b \mid a W) \geq \tau_{2}$, and $P(b \mid a W) / P(b \mid W) \in\left(0 . . \tau_{3}\right] \cup\left[\tau_{4} . .+\infty\right) . \quad$ Alternatively, contexts are all the substrings $a W$ of $T$ with high Kullback-Leibler divergence between the probability distribution of the characters that follow $a W$, and the probability distribution of the characters that follow $W[15,62,14]$, i.e. $\sum_{b \in[1 . . \sigma]} f(a W b) \log (P(b \mid a W) / P(b \mid W)) \geq \tau$ for a positive $\tau$. KL divergence is sometimes 
replaced by the squared $L_{1}$ norm [15], or by any $p$-norm, again using a positive threshold. In yet other variants (e.g. [42, 61, 62]), a substring $W$ of $T$ is called unstable and it is used as a context if it has high entropy compared to all its left-extensions, i.e. if $f(W) H(W)-$ $\sum_{a \in[1 . . \sigma]} f(a W) H(a W) \geq \tau$, where the entropy $H(W)$ equals $-\sum_{b \in[1 . . \sigma]} P(b \mid W) \log P(b \mid W)$ and $\tau$ is a positive threshold. This can be extended to longer left-extensions of a user-specified length. Alternatively, one can use the shortest stable suffix of the current history as a context [61].

\subsection{Variable-order Markov models in small space}

To keep the paper self-contained, we summarize here the key ideas of $[10$, Section 6]. It can been shown that the statistical criteria for choosing contexts in Section 2.2.2 can only select either a maximal repeat, or the left-extension by one character of a maximal repeat (including possibly strings that occur just once in $T$ ). In Section 1 of the supplement we show that such property holds also for a class of IMMs that have been widely used in gene identification [46]. The algorithm in [10] supports any selection criterion in which contexts are maximal repeats or left-extensions thereof: the context tree of $T$ is then the subgraph of the extended suffix-link tree SLT* $T_{T}^{*}$, defined by the following procedure: starting from every node of SLT $T_{T}^{*}$ that corresponds to a context, recursively follow reverse Weiner links up to the root of $\mathrm{SLT}_{T}^{*}$, marking all nodes met in the process.

Our index on $T$ consists of $\overline{\mathrm{BWT}}_{T}$ and the topologies of $\overline{\mathrm{ST}}_{T}$ and $\mathrm{SLT}_{T}$. We represent $\overline{\mathrm{BWT}}_{T}$ as a wavelet tree that supports Weiner links in $O(\log \sigma)$ time, and we use standard compact data structures to support constant-time queries on the balanced parentheses representations of $\overline{\mathbf{S T}}_{T}$ and $\mathrm{SLT}_{T}$ (see Section 9 in the supplement). Let $\operatorname{idSLT}(v)$ be the position of node $v$ of SLT in the preorder traversal of SLT. We build a bitvector $\operatorname{mrSLT}[1 . . p]$, with a bit associated to each of the $p$ nodes of SLT, such that $\operatorname{mrSLT}[\operatorname{idSLT}(v)]=1$ iff $v$ is a maximal repeat. Similarly, let idST $(v)$ be the position of node $v$ of
$\overline{\mathrm{ST}}$ in the preorder traversal of $\overline{\mathrm{ST}}$. We build another bitvector $\operatorname{mrST}[1 . . q]$, with a bit for each of the $q$ nodes of $\overline{\mathrm{ST}}$, such that $\operatorname{mrST}[\operatorname{idST}(v)]=1$ iff $v$ is a maximal repeat. We index mrSLT to support select queries, and mrST to support rank and select queries. Since SLT is a subdivision of the subgraph of $\overline{\mathrm{ST}}$ induced by maximal repeats, the $i$-th one in mrSLT and the $i$-th one in mrST correspond to the same maximal repeat. Thus, if node $v$ is a maximal repeat and if we know $\operatorname{idST}(v)$, we can compute the length of $\ell(v)$ by going to the node $v^{\prime}$ in $\operatorname{SLT}$ with $\operatorname{idSLT}\left(v^{\prime}\right)=$ select $(\operatorname{mrSLT}, 1, \operatorname{rank}(\operatorname{mrST}, 1, \operatorname{idST}(v)))$ and by computing the depth of $v^{\prime}$ in the topology of SLT. In other words, SLT can be seen as a data structure that stores the lengths of all maximal repeats as tree depths. Finally, we include in the index a bitvector context[1..p] such that $\operatorname{context}[\operatorname{idST}(v)]=1$ iff node $v$ in $\overline{\mathrm{ST}}$ is the locus of a context, and a lowest marked ancestor data structure [57] to move in constant time from any node in the topology of $\overline{\mathrm{ST}}$ to its lowest ancestor that is as context.

We summarize how to implement just one of the scoring methods of Section 2.2.1, namely, starting from the longest context $W$ that ends at position $i$ of the query $S$, and using the PPM recursive scoring $\tilde{P}(S[i+1] \mid W)=P(S[i+1] \mid W)$. $(1-\hat{P}(W))$ if $W$ is a context that emits $S[i+1]$, and otherwise $\tilde{P}(S[i+1] \mid W)=\hat{P}(W) \cdot \tilde{P}(S[i+$ 1]|W[2..|W|]), where $\hat{P}(W)$ is either a constant, or a function of $f(S[i+1]), f(W), \sigma, \sigma^{r}(W)$ or $\sigma^{r}(W, 1)$. Recursion stops at the longest context that ends at position $i$ and emits $S[i+1]$. Section 4 details other variants.

The algorithm maps the process of scoring every position $i$ of $S$ onto computing the matching statistics value MS $[i]$, keeping the invariant of knowing, for each $i, \mathrm{MS}[i]$ itself, and the locus of the reverse of $S[i-\mathrm{MS}[i]+1 . . i]$ in $\overline{\mathrm{ST}}$. The actual MS array is never fully present in memory, since MS $[i]$ values are computed on the fly. Assume that contexts are a subset of the maximal repeats of $T$. We scan $S$ from left to right, issuing Weiner link and parent operations on $\overline{\mathrm{ST}}$ as done by standard matching statistics algorithms (see e.g. $[38,9]$ ): such operations can be implemented using just $\overline{\mathrm{BWT}}$ and the topology of $\overline{\mathrm{ST}}$. 
Assume that, after taking a sequence of successful Weiner links from the first position of $S$, we are currently at position $i$ in $S$. Using the topology of $\overline{\mathrm{ST}}$, we convert the interval of the reverse of $W=S[1 . . i]$ in BWT into the idST value of its locus $v$ in $\overline{\mathrm{ST}}$. Note that the label of $v$ is either the reverse of $W$, or the reverse of $X W$, where $X$ is a nonempty string. We then check whether $\operatorname{context}[\operatorname{idST}(v)]=1$. If this is true, then we can measure $|\ell(v)|$ in constant time since $v$ is a maximal repeat, and if it is equal to $i$ we know that $W$ is the longest context that ends at position $i$. If $|\ell(v)|>i$, or if context $[\operatorname{idST}(v)] \neq 1$, we move in constant time to the lowest ancestor $v^{\prime}$ of $v$ that is marked as a context: its label is clearly the longest context that ends at $i$, and the length of its label is necessarily smaller than $i$. We compute the emission probability of character $S[i+1]$ by taking the Weiner link with character $S[i+1]$ from $v^{\prime}$. If such Weiner link exists, or if no such Weiner link exists and we choose not to back off to shorter contexts, we are done. Otherwise, we iteratively issue parent operations from $v^{\prime}$, checking whether the nodes we meet are contexts and if they have a Weiner link by character $S[i+1]$. If this is the case, recursion stops. Otherwise, it continues, and since $v^{\prime}$ is a context, all the nodes we meet are maximal repeats and we can measure the length of their labels, thereby implementing the backoff formula. Once we have assigned a score to position $i+1$ of $S$, we continue as in a standard matching statistics algorithm: we keep increasing $i$ and issuing a Weiner link with character $S[i+1]$ from the locus of the reverse of $S[1 . . i]$ in $\overline{\mathrm{ST}}$. When a Weiner link fails with character $S[i+1]$, we keep issuing parent operations on ST until we reach a node with a Weiner link labeled by $S[i+1]$ : the label of such node is a maximal repeat of $T$, its length is $\operatorname{MS}[i]$, and we can measure such length using SLT: we have thus re-established the invariant for the new value of $i$, and we can repeat the whole process. Just minor modifications are required when contexts are left-extensions by one character of maximal repeats: see Section 2 in the supplement for details. It can be shown that all the operations of the algorithm, including those in the recursive backoff, map onto operations performed by a matching statistics algorithm, and this number is $O(|S|)$; each operation takes $O(\log \sigma)$ time, since Weiner links are the bottleneck.

\section{Smaller variable-order Markov models}

Recall that the algorithm in Section 2.3 works for contexts that are maximal repeats or leftextensions thereof. In repetitive strings, the number of maximal repeats can be significantly smaller than the number of suffix tree nodes [11], thus one could try to shrink the size of the data structures to a quantity related to the number of maximal repeats of the training data. We first show how to do this for computing $\mathrm{MS}_{S, T}$ :

Lemma 1. $\mathrm{MS}_{S, T}$ can be computed using data structures of size proportional to the number of left extensions of maximal repeats of $T$.

Proof. Let $V$ be the set of nodes of $\overline{\mathrm{ST}}_{T}$, let $M \subset V$ be the set of nodes that correspond to maximal repeats, and let $L \subset V$ be the set of leaves. We replace the topology of $\overline{\mathrm{ST}}$ with the topology of the subgraph $G$ of $\overline{\mathrm{ST}}$ induced by $M \cup L$, then we collapse into the same leaf every maximal interval of leaves of $G$ that are consecutive in preorder and that have the same parent in $M$. The size of $G$ is clearly upper bounded by the number of left extensions of maximal repeats of $T$. We use a bitvector leafToMaxrep $[1 . .|T|]$ to mark such intervals of leaves in $\overline{\mathrm{BWT}}_{T}$, then we run-length compress it, i.e. we represent every maximal substring of the same character, as one occurrence of the character followed by the length of the substring (see e.g. [55] for more details). We also run-length encode the bitvector that represents open and closed parentheses in the topology of $\mathrm{SLT}_{T}$ : a maximal run of open (or closed) parentheses corresponds to a unary path in SLT, i.e. to an edge between two maximal repeats. Finally, we run-length encode mrSLT and BWT: the latter can be shown to take a number of words that is upper bounded by the number of left extensions of maximal repeats [11]. Note that we don't need bitvector mrST, since all nodes of $G$ are maximal repeats except for the leaves. 
We compute $\mathrm{MS}_{S, T}$ by scanning $S$ from left to right: we use the run-length compressed BWT to decide whether a Weiner link is successful and to update the BWT interval of the current match. When a Weiner link by character $c$ fails from the current interval in BWT, which corresponds to a node $v$ in $\overline{\mathrm{ST}}$, we know that the lowest ancestor of $v$ that has a Weiner link labelled by $c$ must be a maximal repeat. Thus, we use bitvector leafToMaxrep to move in constant time to the interval of the lowest ancestor of $v$ that is a maximal repeat, and we try the Weiner link again from there. We can measure the string length of this ancestor, and of all its ancestors, since they are maximal repeats.

The probability of $S$ according to the VOMM of $T$ can be computed in a similar way. Indeed, we can use Lemma 1 to maintain, for each position $i$ of $S$, the value MS $[i]$ and the BWT interval of the matching statistics string $W=S[i-\mathrm{MS}[i]+1 . . i]$. Let $v$ be the locus of $\bar{W}$ in $\overline{\mathrm{ST}}$. If contexts are maximal repeats, we use bitvector leafToMaxrep to move to the lowest ancestor of $v$ that is a maximal repeat (which might be $v$ itself). If contexts are left extensions of maximal repeats, we collapse leaves in $G$ in a slightly different way: rather than collapsing into the same leaf all the leaves in a maximal preorder interval with the same lowest maximal repeat ancestor in $\overline{\mathrm{ST}}$, we collapse into the same leaf all leaves in the subtree of $\overline{\mathrm{ST}}$ rooted at the same locus of a left-extension of a maximal repeat. This version of $G$ still takes space proportional to the number of left-extensions of maximal repeats. We build leafToMaxrep correspondingly, and we build and run-length compress bitvectors mrSLT and context

These algorithms can benefit from few additional pruning strategies. For example, when computing MS with Lemma 1, one could further prune the topology of $\overline{\mathrm{ST}}$ (but not of SLT) by keeping just the internal nodes of $\overline{\mathrm{ST}}$ that have a Weiner link that is not present in at least one of their children, and by marking in a bitvector the corresponding nodes in SLT. This is because, when a Weiner link with character $S[i+1]$ fails from the current matching statistics string
$W$, the longest suffix of $W$ that has a Weiner link labelled by $S[i+1]$ corresponds to a node of $\overline{\mathrm{ST}}$ such that its child in the path to the locus of $W$ does not have a Weiner link labelled by $S[i+1]$. The internal nodes of $\overline{\mathrm{ST}}$ that are kept after this pruning are precisely the maximal repeats that are the infix of a minimal absent word of $T$, where a minimal absent word (MAW) of $T$ is a string $W$ that does not occur in $T$, but such that every proper substring of $W$ occurs in $T$ [20]. This pruning applies also to the scoring algorithm, if we just score character $S[i+1]$ with its emission probability from the matching statistics at position $i$, as done e.g. by [33]. Moreover, when contexts are left-extensions of maximal repeats, when the scoring function uses just the longest context that ends at the current position, and when the length of such context does not affect the score, it is easy to see that one can avoid storing the topology of SLT and the bitvectors to commute between $\overline{\mathrm{ST}}$ and SLT altogether.

A second way of reducing the size of our data structures consists in taking advantage of upper bounds on the length of contexts, which are frequently used in applications $[18,45,46]$. We focus here on using such constraint in matching statistics, leaving the immediate application to VOMM scores to the reader. Given an integer $\tau$, we call length-thresholded matching statistics $\mathrm{MS}_{S, T, \tau}[1 . .|S|]$ an array such that $\mathrm{MS}_{S, T, \tau}[i]$ equals $\mathrm{MS}_{S, T}[i]$ if $\mathrm{MS}_{S, T}[i] \leq \tau$, and it equals -1 otherwise.

Lemma 2. We can compute $\mathrm{MS}_{S, T, \tau}$ using the setup of Lemma 1, but replacing the topologies of $\overline{\mathrm{ST}}_{T}$ and of $\mathrm{SLT}_{T}$ with subgraphs induced by maximal repeats of $T$ of length at most $\tau$, and by their left extensions.

Proof. We proceed as in Lemma 1, but rather than considering the subgraph $G$ of $\overline{\mathrm{ST}}$ induced by $M \cup L$, we consider the subgraph $G^{\prime}$ of $\overline{\mathrm{ST}}$ induced by $M_{\tau} \cup L$, where $M_{\tau}$ is the set of maximal repeats of length at most $\tau$, and of all their children. We collapse onto the same leaf all the consecutive leaves of $G^{\prime}$ that have the same parent in $G^{\prime}$. We also consider the subgraph of SLT induced by maximal repeats of length at most 
$\tau$. We maintain the following invariant. At every position $i$ of $S$, we know the value of $\mathrm{MS}_{\tau}[i]$ and: (1) if $\mathrm{MS}_{\tau}[i] \neq-1$, we know the interval of $W_{i}=S[i-\mathrm{MS}[i]+1 . . i]$ in $\overline{\mathrm{BWT}}$; (2) if $\mathrm{MS}_{\tau}[i]=-1$, we know the interval in BWT of a (not necessarily proper) suffix of $W_{i}$ of length greater than $\tau$. We show that we can compute $\mathrm{MS}_{\tau}[i]$ for every $i$ while maintaining such invariant.

Assume that at some position $i$ of $S$ we are in case (2), and let $v$ be the (unknown) locus of $W_{i}$ in the full $\overline{\mathrm{ST}}$. If $v$ can be extended to the right by $S[i+1]$, then $\mathrm{MS}_{\tau}[i+1]=-1$, the right extension is possible also from the BWT interval of the suffix of $W_{i}$ that we have, and performing such extension maintains the invariant that we know the interval in BWT of a suffix of $W_{i+1}$ of length bigger than $\tau$. If $v$ cannot be extended to the right with character $S[i+1]$, but there is a Weiner link labelled by character $S[i+1]$ from the interval that we have, it means again that $\mathrm{MS}_{\tau}[i+1]=-1$, and that $v$ is a descendant of the locus in $\overline{\mathrm{ST}}$ of our interval, thus taking the Weiner link preserves the invariant that we know the interval in BWT of a suffix of $W_{i+1}$ of length bigger than $\tau$. Finally, if $v$ cannot be extended to the right with character $S[i+1]$, and if the interval we have cannot be extended to the right with $S[i+1]$ either, we use leafToMaxrep to convert the BWT interval that we have into an internal node $v^{\prime}$ of $G^{\prime}$. Note that $\left|\ell\left(v^{\prime}\right)\right| \geq \tau$, but $v^{\prime}$ is not necessarily a maximal repeat. If $v^{\prime}$ does not have a Weiner link labeled by $S[i+1]$ either, then $\mathrm{MS}_{\tau}[i+1] \neq-1$, and we issue parent operations in the topology of $G^{\prime}$ that are identical to the parent operations we would have issued from $v$ in the full $\overline{\mathrm{ST}}$, reaching eventually the same maximal repeat $w$ that has a Weiner link labeled by $S[i+1]$ and whose length is at most $\tau$. Otherwise, if $v^{\prime}$ has a Weiner link labeled by $S[i+1]$, then $\mathrm{MS}_{\tau}[i+1]=-1, v^{\prime}$ is necessarily a maximal repeat, and $w$ is either $v^{\prime}$ itself or a descendant of $v^{\prime}$. Thus, by extending $v^{\prime}$ (rather than $w$ ) to the right by $S[i+1]$, we maintain the invariant that we know the interval in BWT of a suffix of $W_{i+1}$ of length greater than $\tau$.

Finally, assume that at some position $i$ of $S$ we are in case (1). As long as $W_{i}$ can be extended to

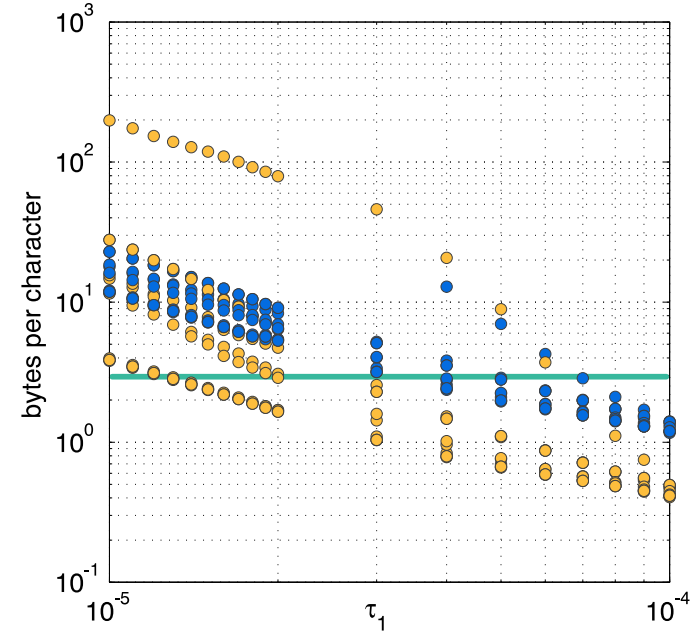

Figure 1: Size of trie representations of VOMMs as a function of $\tau_{1}$ (the minimum empirical probability of a context) in the four-thresholds context selection criterion of Section 2.2.2, over ten non-repetitive concatenations of proteins of length one million each. Decreasing $\tau_{1}$ implies increasing the number of contexts. Blue circles: the probabilistic suffix tree code in [5]; orange circles: the SPST code in [31]. Our implementation has a small variance, so it is represented just as a green line (averages) rather than as circles.

the right by $S[i+j]$, we take a Weiner link and we update the value of $\mathrm{MS}_{\tau}[i+j]$ for increasing $j$. Note that at each step we know the interval of MS $[i+j]$, even when, for some $j$, a right extension leads to a string that is longer than $\tau$. Finally, for some $j$, a right extension might not be possible. If $\mathrm{MS}[i+j-1]>\tau$ we do the same operations as in case (2), preserving the invariant. If $\mathrm{MS}[i+j-$ $1] \leq \tau$, the current interval contains a range of leaves of $G^{\prime}$ : we use leafToMaxrep to reach the lowest common ancestor of such leaves, which is necessarily a maximal repeat, and we continue from there.

Since frequency decreases monotonically with tree depth on a suffix tree, Lemma 2 can be adapted to compute the frequency-thresholded matching statistics $\mathrm{MS}_{S, T, \tau}[1 . .|S|]$, which is an array such that $\mathrm{MS}_{S, T, \tau}[i]$ equals $\mathrm{MS}_{S, T}[i]$ if $f_{T}\left(S\left[i-\mathrm{MS}_{S, T}[i]+1 . . i\right]\right) \geq \tau$, and it equals -1 otherwise. 


\section{Variants and extensions}

We sketch the main ideas for supporting the variety of VOMMs and IMMs in Sections 2.2.1 and 2.2.2, omitting some technical details for brevity. We are not interested in achieving the fastest algorithm for each variant, but in showing that all variants can be implemented with just minor modifications to the non-pruned data structures of Section 2.3, and possibly to the pruned data structures of Section 3.

We already described in Section 3 how to support the scoring of [33]. To support the scoring scheme that uses the shortest right-deterministic suffix of $W_{i}$ as a context for position $i[59,19]$, it suffices to mark as contexts all nodes of $\overline{\mathrm{ST}}$ that are not maximal repeats, and such that their parent is a maximal repeat, and to proceed as in the case in which contexts are left-extensions of maximal repeats. Thus, this strategy can be implemented also with maximal repeat pruning. Recall that one of the statistical criteria used in Section 2.2.2 to mark a substring $W$ as a context, checks whether the entropy of the emission probabilities from $W$ is significantly larger than the entropy from the left-extensions of $W$. To support the symmetrical criterion of using the shortest stable suffix of $W_{i}$, i.e. the shortest suffix that has small entropy compared to its leftextensions [61], it suffices to mark the loci of all stable substrings without a stable ancestor in $\overline{\mathrm{ST}}$, and to jump to one such locus from the locus of a matching statistics string $W_{i}$. This strategy can thus be implemented with maximal repeat pruning as well. To enforce that no suffix of a context is itself a context $[31,54,15]$, we can just unmark all nodes with a marked ancestor in $\overline{\mathrm{ST}}$. Enforcing that all prefixes of a context must be contexts (e.g. to make the Markov model memoryless: see e.g. [34]) does not guarantee that contexts are a subset of maximal repeats or of their left extensions. However, we can enforce that all prefixes of a context are suffixes of a context, by just adding leftmost maximal repeats or their left extensions to the set of contexts.

During construction we can compute the number of distinct characters in the BWT interval of every context, and we can store such numbers in an array of $O(m \log \sigma)$ bits, where $m$ is the number of contexts. Given a query $S$, we can then compute the average number of right-extensions of all contexts that are used for scoring a position of $S$ [37], by just accessing the array. The same approach can be used to access $\sigma^{\ell}(W), \sigma^{r}(W)$ or $\sigma^{r}(W, 1)$ for a context $W$ at query time: together with $f(W)$ and with the total frequency of single characters, such quantities are enough to implement a number of probability smoothings.

The recursive interpolation schemes of Section 2.2.1 can be implemented by iteratively taking parent operations in $\overline{\mathrm{ST}}$, as described in Section 2.3. If the user provides an arbitrary weight function based on string length, we can implement the weighted mixing of suffix contexts by storing an array of prefix sums of log-weights: such array might not take too much space in practice if the string length of the longest edge of $\overline{\mathrm{ST}}$ is small. If weights are constant, or if they are just a function of frequency and of the number of distinct characters to the right of a suffix of a context, they don't change along an edge of $\overline{\mathrm{ST}}$. If they are a function of the length of a suffix of a context, and if such function is known in closed form [23], the contribution of all weighted probabilities along any edge of $\overline{\mathrm{ST}}$ might even be computable in constant time. If we need the maximum emission probability among all suffixes of the longest context that are themselves contexts [37], we can just iterate calls to the lowest marked ancestor data structure. We can implement the IMM in Section 1 of the supplement in a similar way, by computing the values of $p(W)$ during construction, and storing them in an array with one entry per left extension of a maximal repeat. We can then mark as context the locus $v$ in $\overline{\mathrm{ST}}$ of all strings $a W$ such that $f(a W)$ is smaller than the given threshold and $W$ is a maximal repeat. During scoring, we iteratively move from one context to the other using the lowest marked ancestor data structure.

To compute the backoff function $\beta(W, a)$ of Section 2.2.1, we need to compute $\sigma^{\ell}(W[2 . .|W|] a)$ for a context $W$ [29]. Since contexts are maximal repeats, or left-extensions by one character of maximal repeats, we can decide whether $\overline{W[2 . .|W|]}$ ends in the middle of 


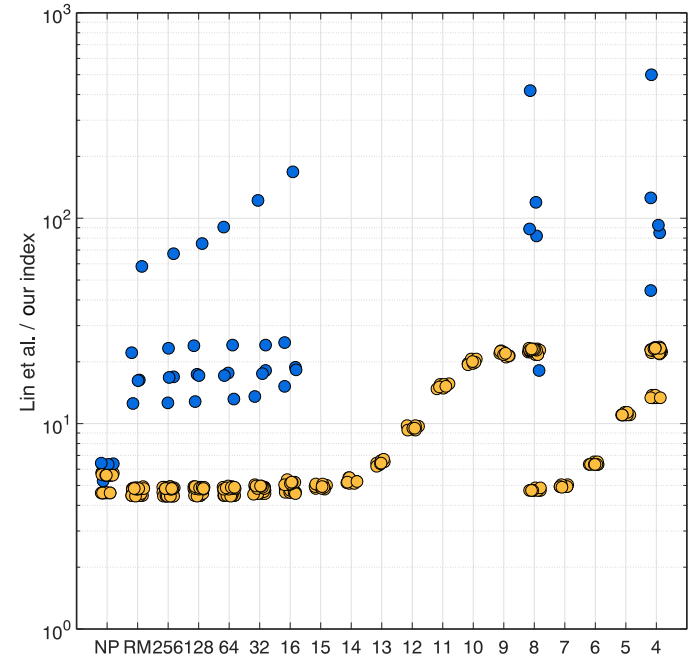

Figure 2: The size of our index compared to the size of [33], for strings of length at most 80 million. Orange circles: non-repetitive datasets; blue circles: repetitive datasets. See the text for more details. Note that the vertical axis has a logarithmic scale. Circles of the same color trace multiple curves because they belong to different datasets.

an edge of ST or not. If $\overline{W[2 . .|W|]}$ ends in the middle of an edge, then $\sigma^{\ell}(W[2 . .|W|] a)$ is either one or zero, depending on whether a Weiner link with character $a$ exists from the locus of $\overline{W[2 . .|W|]}$ in ST. If $\overline{W[2 . .|W|]}$ ends at a node of $\overline{\mathrm{ST}}$, and if a Weiner link exists from such node with label $a$, then we need to count the number of distinct characters to the left of $W[2 . .|W|] a$, i.e. the number of children of the locus of $\overline{W[2 . .|W|]}$ that have a Weiner link by character a. A similar method can be used to implement the Kneser-Ney smoothings in $[52,53,51,30]$. Section 3 in the supplement describes how to compute some globals statistics on $T$ using the same setup of data structures.

\section{$5 \quad$ Experimental results}

Our $\mathrm{C}++$ implementation is based on the SDSL library [26] and is sequential: engineering it to use parallel threads falls outside the scope of this paper, and is discussed in Section 7 of the supplement. See Section 4 in the supplement for more implementation details.

We study the size and composition of our indexes in a number of datasets whose content and scale approximate current and possibly future applications of VOMMs. We focus on large datasets that were never tackled in previous works, and specifically: (1) The concatenation of all sequences in the latest reference assembly of the human genome in NCBI (5.9 billion characters). (2) The concatenation of all the approximately 8600 bacterial genomes currently in NCBI (34 billion characters). (3) The repetitive collections of bacterial genomes in the Pizza\&Chili corpus $^{1}$ : these files range from approximately 100 to 400 million characters, and contain the concatenation of 23 genomes of $E$. coli, of approximately 35 genomes of $S$. paradoxus and of $S$. cerevisiae, and of approximately 78 thousand genomes of $H$. influenzae. (4) The concatenation of all non-redundant protein sequences in the NCBI RefSeq database ([41], 29 billion characters). (5) A repetitive dataset of proteins, built from the bacterial protein clusters in NCBI as described in Section 5 of the supplement (70 million characters). (6) The concatenation of all Illumina reads in a WGS metagenomic stool sample from the Human Microbiome Project ([36], 28 billion characters). (7) A prefix of the concatenation of all reads from a human individual in the Illumina Platinum project ${ }^{2}$ (33 billion characters). See Section 5 in the supplement for additional details on the datasets. The strings we use are long, but they are far from the longest ones that can be indexed by our implementation on a standard server with e.g. one terabyte of RAM (approximately 80 billion characters). For each string, we build: (1) the plain version of our index, with no pruning; (2) a version of the index in which topologies are pruned based on maximal repeats, and the BWT and the bitvectors are run-length encoded; (3) a version of (2), further pruned at depths that are powers of two. For concreteness, we select contexts using just the four-thresholds criterion of Section 2.2.2: see Section 5 in the supplement for details.

\footnotetext{
${ }^{1}$ http://pizzachili.dcc.uchile.cl/repcorpus.html

${ }^{2}$ https ://emea.illumina.com/platinumgenomes.html
} 
The size and composition of our indexes are summarized in Figure 3. The plain index takes approximately three bytes per character, with data structures for $\overline{\mathrm{ST}}$ taking up the largest fraction of the total size, and bitvector context taking up approximately $16 \%$ of the total. Compression by maximal repeats saves between $30 \%$ and $50 \%$ of the size of the index in datasets that are not very repetitive, but it saves approximately $90 \%$ in repetitive collections like $S$. cerevisiae. In repetitive collections, bitvector leaf ToMaxrep takes approximately between $15 \%$ and $30 \%$ of the total size of the compressed index. Note that leafToMaxrep consists mostly of zeros, or mostly of ones, if there is little pruning or a lot of pruning, so it compresses well with run-length encoding. If there is a moderate amount of pruning, the distribution of zeros and ones is more even, and compression does not work as well. Further pruning by context length can shrink the compressed index to half of its size and beyond, but the the depth at which significant space reduction occurs depends on the dataset.

In large collections of short strings, like proteins or reads, or in datasets that are not very repetitive, maximal repeats are short and potentially few: rather than storing the topology of SLT and the bitvectors to move from $\overline{\mathrm{ST}}$ to SLT, it might be more space-efficient in practice to encode the length of every maximal repeat in preorder. We experiment with the simplest possible scheme, i.e. storing each depth with a fixed number of bits that is just large enough to represent the largest length of a maximal repeat; we discuss more advanced methods in Section 8 of the supplement. Even with our simple encoding, explicit storage takes always less space than storing SLT, except in the human genome and in the concatenation of all bacterial genomes from NCBI (Figure 6 in the supplement). In the collection of E. coli genomes, explicit storage takes space comparable to the compressed SLT; otherwise, it allows one to save from $12 \%$ to $40 \%$ of the space of the compressed SLT.

Our VOMM implementation has marked advantages with respect to existing ones. As expected, the size of the index built by [5] depends on the number and length of contexts, so it produces smaller indexes than ours for restrictive settings of the thresholds. However, contrary to our data structure, index size blows up when thresholds are set to less restrictive values, becoming 10 times bigger than ours, or more (Figure 1): the limited scalability of pointerbased representations is the very motivation for research on space-efficient VOMMs. The code of [33], based on the suffix array, cannot index strings of length bigger than 80 million characters in practice, so we have to study its behavior on substrings of such length of all our datasets. Specifically, we take random substrings of the human genome, of the concatenation of all bacterial genomes, and of the concatenation of all non-redundant proteins, yielding non-repetitive strings; we use the full repetitive proteins dataset; and we take prefixes of the Pizza\&Chili repetitive strings, which should still yield repetitive strings (albeit possibly less repetitive than the original). For non-repetitive strings, our index is between 3 and 4 times smaller than the competitor, and it becomes up to 11 times smaller after length pruning (Figure 2 ). In repetitive strings, our compressed index is from 10 to 60 times smaller than the competitor, and it becomes up to 500 times smaller after depth pruning (Figure 2). We remark again that such space savings should not be taken as upper bounds, since we are experimenting with short strings that might be less repetitive than the original datasets. We also note that, in addition to being larger, [33] supports just one type of score. The index in $[53]^{3}$ takes from 3 to 3.3 bytes per character, regardless of the compressibility of the input. After removing precomputed counts used to speed up queries, the index takes between 2.6 and 3.1 bytes per character, which should approximate the size of the index in [52] and is comparable to our non-pruned data structure. Recall, however, that the competitor supports just an IMM with Kneser-Ney smoothing.

Optimizing index construction is outside the scope of this paper. However, our sequential construction algorithms are reasonably fast and space-efficient in practice: see Section 6 in the

\footnotetext{
${ }^{3}$ https://github.com/eehsan/cstlm
} 
supplement for details and for a comparison to the competitors. Maximizing the speed of scoring functions is also outside the scope of this paper. Studying scoring time is interesting, however, since we expect to be paying our space savings in this currency. We report a detailed analysis of scoring time in Section 7 of the supplement. Here we just mention that non-recursive scoring on our index with maximal repeats pruning is indeed approximately 5-6 times slower than the same scoring with our non-pruned index; however, pruning the compressed index further by maximum length of a context makes scoring up to 2 times faster. Our non-pruned index is between 60 and 35 times slower than the trie-based index in [5], and it is between 3 and 12 times slower than [33] to compute the specific score of the competitor, depending on the dataset.

\section{Acknowledgements}

We thank V. Mäkinen for guidance, E. Shareghi for help with the code of [53], and O. Gonzalez and P. Steinbach for help with the MPI-CBG cluster.

\section{References}

[1] Alberto Apostolico and Gill Bejerano. Optimal amnesic probabilistic automata or how to learn and classify proteins in linear time and space. Journal of Computational Biology, 7(3-4):381-393, 2000.

[2] Dror Baron and Yoram Bresler. An $O(n)$ semipredictive universal encoder via the BWT. IEEE Transactions on Information Theory, 50(5):928-937, 2004.

[3] Ron Begleiter, Ran El-Yaniv, and Golan Yona. On prediction using variable order Markov models. Journal of Artificial Intelligence Research, 22:385-421, 2004.

[4] Gill Bejerano. Automata learning and stochastic modeling for biosequence analysis. PhD thesis, Hebrew University of Jerusalem, 2003.
[5] Gill Bejerano. Algorithms for variable length Markov chain modeling. Bioinformatics, 20(5):788-789, 2004.

[6] Gill Bejerano, Yevgeny Seldin, Hanah Margalit, and Naftali Tishby. Markovian domain fingerprinting: statistical segmentation of protein sequences. Bioinformatics, 17(10):927-934, 2001.

[7] Gill Bejerano and Golan Yona. Modeling protein families using probabilistic suffix trees. In Proceedings of the third Annual International Conference on Computational Molecular Biology, pages 15-24. ACM, 1999.

[8] Gill Bejerano and Golan Yona. Variations on probabilistic suffix trees: statistical modeling and prediction of protein families. Bioinformatics, 17(1):23-43, 2001.

[9] Djamal Belazzougui and Fabio Cunial. Indexed matching statistics and shortest unique substrings. In International Symposium on String Processing and Information Retrieval, pages 179-190. Springer, 2014.

[10] Djamal Belazzougui and Fabio Cunial. A framework for space-efficient string kernels. Algorithmica, pages 1-27, 2017.

[11] Djamal Belazzougui, Fabio Cunial, Travis Gagie, Nicola Prezza, and Mathieu Raffinot. Composite repetition-aware data structures. In Annual Symposium on Combinatorial Pattern Matching, pages 26-39. Springer, 2015.

[12] Arthur Brady and Steven L Salzberg. Phymm and PhymmBL: metagenomic phylogenetic classification with interpolated Markov models. Nature Methods, 6(9):673676, 2009.

[13] Thorsten Brants, Ashok C Popat, Peng Xu, Franz J Och, and Jeffrey Dean. Large language models in machine translation. In Proceedings of the 2007 Joint Conference on Empirical Methods in Natural Language Processing and Computational Natural Language Learning (EMNLP-CoNLL), 2007. 

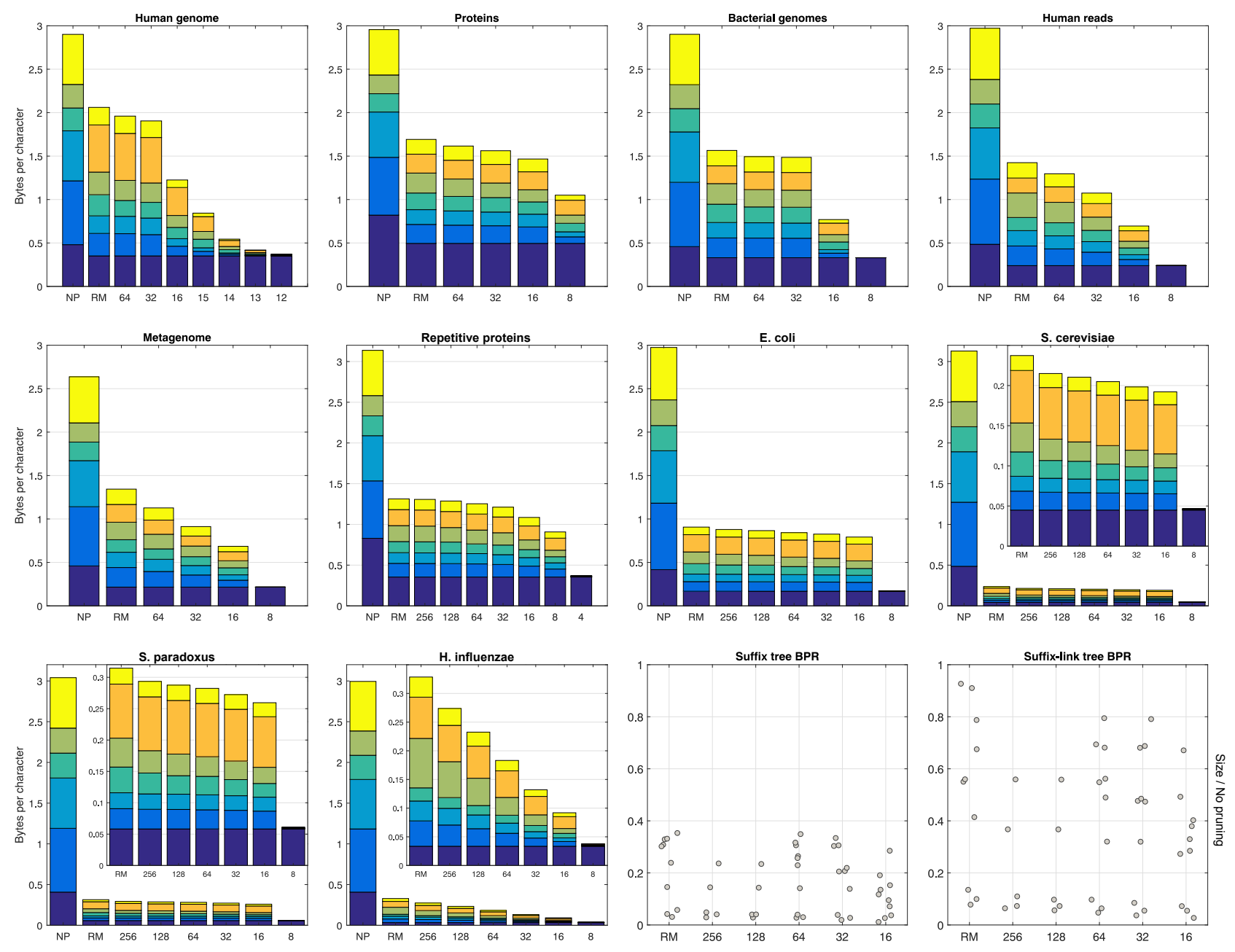

$\square$ BWT

$\square$ ST bpr

$\square$ ST maxrep $\square$ SLT bpr

$\square$ SLT maxrep $\square$ pruning marks

$\square$ ST contexts

Figure 3: Size and composition of our indexes on large datasets. NP: no pruning; RM: run-length encoding (RLE) and maximal repeat pruning; numbers: RLE, maximal repeat pruning, and depth pruning. Pruning marks: all data structures associated with pruning $\overline{\mathrm{ST}}$ and SLT, including leafToMaxrep. The two dot plots on the bottom-right panels show the size of the balanced parentheses representations of $\overline{\mathrm{ST}}$ and SLT, divided by their size without pruning (bitvectors context, mrSLT and mrST have similar plots). 
[14] Peter Bühlmann. Model selection for variable length Markov chains and tuning the context algorithm. Annals of the Institute of Statistical Mathematics, 52(2):287-315, 2000 .

[15] Peter Bühlmann, Abraham J Wyner, et al. Variable length Markov chains. The Annals of Statistics, 27(2):480-513, 1999.

[16] Suzanne Bunton. Semantically motivated improvements for PPM variants. The Computer Journal, 40(2_and_3):76-93, 1997.

[17] Stanley F Chen and Joshua Goodman. An empirical study of smoothing techniques for language modeling. Computer Speech 83 Language, 13(4):359-394, 1999.

[18] John Cleary and Ian Witten. Data compression using adaptive coding and partial string matching. IEEE transactions on Communications, 32(4):396-402, 1984.

[19] John G Cleary and William J Teahan. Unbounded length contexts for PPM. The Computer Journal, 40(2_and_3):67-75, 1997.

[20] Maxime Crochemore, Filippo Mignosi, and Antonio Restivo. Automata and forbidden words. Information Processing Letters, 67(3):111-117, 1998.

[21] Daniel Dalevi, Devdatt Dubhashi, and Malte Hermansson. Bayesian classifiers for detecting HGT using fixed and variable order Markov models of genomic signatures. Bioinformatics, 22(5):517-522, 2006.

[22] Ofer Dekel, Shai Shalev-Shwartz, and Yoram Singer. The power of selective memory: self-bounded learning of prediction suffix trees. In Advances in Neural Information Processing Systems, pages 345-352, 2005.

[23] Ofer Dekel, Shai Shalev-Shwartz, and Yoram Singer. Individual sequence prediction using memory-efficient context trees. IEEE Transactions on Information Theory, 55(11):5251-5262, 2009.
[24] Arthur L Delcher, Kirsten A Bratke, Edwin C Powers, and Steven L Salzberg. Identifying bacterial genes and endosymbiont DNA with GLIMMER. Bioinformatics, 23(6):673-679, 2007.

[25] Arthur L Delcher, Douglas Harmon, Simon Kasif, Owen White, and Steven L Salzberg. Improved microbial gene identification with GLIMMER. Nucleic Acids Research, 27(23):4636-4641, 1999.

[26] Simon Gog, Timo Beller, Alistair Moffat, and Matthias Petri. From theory to practice: plug and play with succinct data structures. In 13th International Symposium on Experimental Algorithms (SEA 2014), pages 326-337, 2014.

[27] Michael J Kearns and Yishay Mansour. A fast, bottom-up decision tree pruning algorithm with near-optimal generalization. In ICML, volume 98, pages 269-277. Citeseer, 1998.

[28] David R Kelley and Steven L Salzberg. Clustering metagenomic sequences with interpolated Markov models. BMC Bioinformatics, 11(1):544, 2010.

[29] Christopher Kermorvant and Pierre Dupont. Improved smoothing for probabilistic suffix trees seen as variable order Markov chains. In European Conference on Machine Learning, pages 185-194. Springer, 2002.

[30] Christopher Kermorvant and Pierre Dupont. Mélanges de chaînes de Markov lissées pour la détection de domaines dans les protéines. In Proceedings des Journées Ouvertes Biologie Informatique Mathématique, 2002.

[31] Florencia G Leonardi. A generalization of the PST algorithm: modeling the sparse nature of protein sequences. Bioinformatics, 22(11):1302-1307, 2006.

[32] Weinan Liao, Jie Ren, Kun Wang, Shun Wang, Feng Zeng, Ying Wang, and Fengzhu 
Sun. Alignment-free transcriptomic and metatranscriptomic comparison using sequencing signatures with variable length Markov chains. Scientific Reports, 6:37243, 2016.

[33] Jie Lin, Donald Adjeroh, and Bing-Hua Jiang. Probabilistic suffix array: efficient modeling and prediction of protein families. Bioinformatics, 28(10):1314-1323, 2012.

[34] Joshua Magarick. Sequential Learning and Variable Length Markov Chains. PhD thesis, University of Pennsylvania, 2016.

[35] Alvaro Martin, Gadiel Seroussi, and Marcelo J Weinberger. Linear time universal coding and time reversal of tree sources via FSM closure. IEEE Transactions on Information Theory, 50(7):1442-1468, 2004.

[36] Barbara A Methé, Karen E Nelson, Mihai Pop, Heather H Creasy, Michelle G Giglio, Curtis Huttenhower, Dirk Gevers, Joseph F Petrosino, Sahar Abubucker, Jonathan H Badger, et al. A framework for human microbiome research. Nature, 486(7402):215, 2012.

[37] Hasan Oğul and Erkan Ü Mumcuoğlu. SVM-based detection of distant protein structural relationships using pairwise probabilistic suffix trees. Computational Biology and Chemistry, 30(4):292-299, 2006.

[38] Enno Ohlebusch, Simon Gog, and Adrian Kügel. Computing matching statistics and maximal exact matches on compressed fulltext indexes. In SPIRE, pages 347-358, 2010 .

[39] Uwe Ohler, Stefan Harbeck, Heinrich Niemann, E Nöth, and Martin G Reese. Interpolated Markov chains for eukaryotic promoter recognition. Bioinformatics (Oxford, England), 15(5):362-369, 1999.

[40] Fernando C Pereira and Yoram Singer. An efficient extension to mixture techniques for prediction and decision trees. Machine Learning, 36(3):183-199, 1999.
[41] Kim D Pruitt, Tatiana Tatusova, and Donna R Maglott. NCBI reference sequence (RefSeq): a curated non-redundant sequence database of genomes, transcripts and proteins. Nucleic Acids Research, 33(suppl_1):D501-D504, 2005.

[42] Jorma Rissanen. A universal data compression system. IEEE Transactions on Information Theory, 29(5):656-664, 1983.

[43] Jorma Rissanen and G Langdon. Universal modeling and coding. IEEE Transactions on Information Theory, 27(1):12-23, 1981.

[44] Eric Sven Ristad and Robert G Thomas. Nonuniform Markov models. In Acoustics, Speech, and Signal Processing, $199 \%$. ICASSP-97., 1997 IEEE International Conference on, volume 2, pages 791-794. IEEE, 1997.

[45] Dana Ron, Yoram Singer, and Naftali Tishby. The power of amnesia: learning probabilistic automata with variable memory length. Machine learning, 25(2-3):117149, 1996.

[46] Steven L Salzberg, Arthur L Delcher, Simon Kasif, and Owen White. Microbial gene identification using interpolated Markov models. Nucleic Acids Research, 26(2):544-548, 1998.

[47] Marcel Schulz. Personal communication, 2018.

[48] Marcel H Schulz, Hugues Richard, and Martin Vingron. Efficient estimation of variable order Markov chains. In Proceedings of the 15th Annual International Conference on Intelligent Systems for Molecular Biology (ISMB) and 6th European Conference on Computational Biology (ECCB), 2007.

[49] Marcel H Schulz, David Weese, Tobias Rausch, Andreas Döring, Knut Reinert, and Martin Vingron. Fast and adaptive variable order Markov chain construction. In International Workshop on Algorithms in Bioinformatics, pages 306-317. Springer, 2008. 
[50] Yevgeny Seldin, Gill Bejerano, and Naftali Tishby. Unsupervised sequence segmentation by a mixture of switching variable memory Markov sources. In $I C M L$, pages $513-520,2001$.

[51] Ehsan Shareghi, Trevor Cohn, and Gholamreza Haffari. Richer interpolative smoothing based on modified Kneser-Ney language modeling. In Proceedings of the 2016 Conference on Empirical Methods in Natural Language Processing, pages 944-949, 2016.

[52] Ehsan Shareghi, Matthias Petri, Gholamreza Haffari, and Trevor Cohn. Compact, efficient and unlimited capacity: language modeling with compressed suffix trees. In Proceedings of the 2015 Conference on Empirical Methods in Natural Language Processing, pages 2409-2418, 2015.

[53] Ehsan Shareghi, Matthias Petri, Gholamreza Haffari, and Trevor Cohn. Fast, small and exact: infinite-order language modelling with compressed suffix trees. Transactions of the Association for Computational Linguistics, 4:477-490, 2016.

[54] Yoram Singer. Adaptive mixture of probabilistic transducers. In Advances in Neural Information Processing Systems, pages 381387, 1996.

[55] Jouni Sirén, Niko Välimäki, Veli Mäkinen, and Gonzalo Navarro. Run-length compressed indexes are superior for highly repetitive sequence collections. In International Symposium on String Processing and Information Retrieval, pages 164-175. Springer, 2008.

[56] Padhraic Smyth et al. Clustering sequences with hidden Markov models. Advances in Neural Information Processing Systems, pages 648-654, 1997.

[57] Shoshana Marcus Dina Sokol. Engineering small space dictionary matching. arXiv preprint arXiv:1301.6428, 2013.
[58] Marc Strous, Beate Kraft, Regina Bisdorf, and Halina Tegetmeyer. The binning of metagenomic contigs for microbial physiology of mixed cultures. Frontiers in Microbiology, 3:410, 2012.

[59] WJ Teahan. Probability estimation for PPM. In In Proceedings NZCSRSC'95., 1995.

[60] Georgios S Vernikos and Julian Parkhill. Interpolated variable order motifs for identification of horizontally acquired DNA: revisiting the Salmonella pathogenicity islands. Bioinformatics, 22(18):2196-2203, 2006.

[61] Marcelo J Weinberger, A Lempe, and Jacob Ziv. A sequential algorithm for the universal coding of finite memory sources. IEEE Transactions on Information Theory, 38(3):1002-1014, 1992.

[62] Marcelo J Weinberger, Jorma J Rissanen, and Meir Feder. A universal finite memory source. IEEE Transactions on Information Theory, 41(3):643-652, 1995.

[63] Frans MJ Willems, Yuri M Shtarkov, and Tjalling J Tjalkens. The contexttree weighting method: basic properties. IEEE Transactions on Information Theory, 41(3):653-664, 1995.

[64] Ian H Witten and Timothy C Bell. The zero-frequency problem: estimating the probabilities of novel events in adaptive text compression. IEEE Transactions on Information Theory, 37(4):1085-1094, 1991. 


\title{
Supplementary material for: \\ "A framework for space-efficient variable-order Markov models"
}

\author{
Fabio Cunial Jarno Alanko Djamal Belazzougui
}

\section{IMMs and maximal repeats}

We show that the IMM in [29] performs a linear combination of the emission probabilities of just the left extensions by one character of maximal repeats. Recall from Section 2.2.1 in the paper that such IMM assigns the following score to observing character $b$ after context $W: \tilde{P}(b \mid W)=\lambda(W) \cdot P(b \mid W)+(1-\lambda(W)) \cdot \tilde{P}(b \mid W[2 . .|W|])$ if $|W|>0$, and $f(b) /|T|$ otherwise. If $f(W)$ is at least a given threshold, then $\lambda(W)$ is set to one and recursion stops. Otherwise, $\lambda(W)$ is set to either zero or $\lambda^{\prime}(W)=c \cdot(1-p(W)) \cdot f(W b)$, where $c$ is a constant and $p(W)$ is the fraction of mass of the $\chi^{2}$ distribution to the right of the Pearson's chi-squared test statistic $x(W)$. Such statistic uses the frequencies of $W a$ as observed frequencies, and the frequencies of $W[2 . .|W|] a$ as expected frequencies, for every $a \in \Sigma$, i.e. $x(W)=f(W) \cdot \sum_{a \in \Sigma^{r}(W[2 . .|W|])}(P(a \mid W)-P(a \mid W[2 . .|W|]))^{2} / P(a \mid W[2 . .|W|])$. The IMM chooses between setting $\lambda(W)$ to zero or to $\lambda^{\prime}(W)$ by comparing $1-p(W)$ to a positive, user-defined threshold. Note that, if $W[2 . .|W|]$ if not left-maximal, $P(a \mid W)$ coincides with $P(a \mid W[2 . .|W|])$ for any $a$, thus $x(W)=0$. Similarly, if $W[2 . .|W|]$ is always followed by character $b$, the summation in $x(W)$ runs just over $b$, and both $P(b \mid W)$ and $P(b \mid W[2 . .|W|])$ are equal to one, thus again $x(W)=0$. When $x(W)=0$, we also have that $p(W)=1,1-p(W)=0$, and $\lambda(W)=0$.

\section{Contexts as left extensions of maximal repeats}

We describe how to modify the algorithm in Section 2.3 of the paper to handle contexts that are left extensions by one character of maximal repeats. Assume again that we mark the locus of all such contexts in the topology of $\overline{\mathrm{ST}}$. After taking a sequence of successful Weiner links from the first position of $S$, assume that we are currently at position $i$ of $S$. We go to the locus $v$ of the reverse of $W=S[1 . . i]$ in $\overline{\mathrm{ST}}$ and, if $v$ is marked, we use the emission probability from $v$ to score $S[i+1]$ : this is equivalent to using the emission probability of the context $S\left[i-\ell\left(v^{\prime}\right) . . i\right]$, where $v^{\prime}$ is the parent of $v$ in $\overline{\mathrm{ST}}$. If $v$ is not marked, we move to its lowest marked ancestor $v^{\prime}$. When we back off from a node $w$ to a node $w^{\prime}$ 
of $\overline{\mathrm{ST}}$, where $w$ does not emit $S[i+1]$ and $w^{\prime}$ if the lowest marked ancestor of $w$ that emits $S[i+1]$, we adapt the algorithm to take into account that now we are considering contexts $S[i-\ell(u) . . i], \ldots, S\left[i-\ell\left(u^{\prime}\right) . . i\right]$, where $u$ is the parent of $w$ and $u^{\prime}$ is the parent of $w^{\prime}$.

\section{Statistics on the training data}

Note that, once we have built our data structures on $T$, we can sample a string $S$ from the VOMM using the scoring algorithm in Section 2.3 of the paper, with just the topology of $\overline{\mathrm{ST}}$, the BWT of the reverse, and the lowest marked ancestor data structure: indeed, we can start from a random context, and iteratively sample a Weiner link at random, based on the emission probabilities of the current context, and then move from the locus of the resulting extension to its lowest ancestor in $\overline{\mathrm{ST}}$ that is a context.

The same setup can be used to compute a number of global statistics on $T$, for example a maximum-likelihood estimate of the order of the fixed-order Markov chain that generated $T$ : this is the value of $k$ that minimizes $-\log \hat{P}_{k}(T)+(\sigma-1) \sigma^{k} \log (|T|-k+1) / 2$, where $\hat{P}_{k}(T)=\prod_{W \in \Sigma^{k}, f(W a)>0}(f(W a) / f(W))^{f(W a)}[14]$. To compute $\hat{P}_{k}(T)$ for a specific value of $k$, it suffices to mark as contexts just the loci of $k$-mers in $\overline{\mathrm{ST}}$, and to score $T$ against its VOMM. The same holds for computing the $k$-th order Markov predictability of $T$, defined as $(1 /(|T|-k)) \cdot \sum_{W \in \Sigma^{k}} \sum_{a \in \Sigma \backslash\{\gamma(W)\}} f(W a)$, where $\gamma(W)=\operatorname{argmax}\{a \in \Sigma: f(W a)>0\}$, for a user-specified range of values of $k$ [15]. This is the fraction of errors while predicting the characters of $T$ using contexts of fixed length $k$, predicting as the next character, at each position of $T$, the character with largest relative frequency with respect to the current $k$-mer.

\section{Details on the implementation}

We build the BWT with the divsufsort library ${ }^{1}$. We use the rank data structure rank_support_v and the code for select operations on bitvectors select_support_mcl from the SDSL library [18], and we represent the topology of ST and of SLT explicitly with balanced parentheses, using bp_support_g. We build most data structures using our own implementation of the bidirectional BWT index ${ }^{2}$, and we use portions of the lz-rlbwt code $^{3}$ from [10] for the run-length-encoded BWT. One could plug in other implementations to build the indexes, for example [2] for the suffix tree topology; we haven't experimented with such alternatives since we do not try to optimize construction. The implementation ships with comprehensive unit tests on randomized strings to ensure correctness.

When comparing our implementation to the code in [4], we turn off computing and storing statistics in the competitor's source, and we compile it with gcc -03 .

\footnotetext{
${ }^{1}$ https://github.com/y-256/libdivsuf sort

${ }^{2}$ https://github.com/jnalanko/BD_BWT_index

${ }^{3}$ https://github.com/nicolaprezza/lz-rlbwt
} 


\section{Details on the datasets}

Our metagenomic stool sample is file SRS301868 at https://portal .hmpdacc.org/files/ 91319e642fdd8a6e3b059cfb058cc4aa, and our human reads from the Illumina Platinum project are from run ERR194146, file ERR194146_1.fastq.gz, from

https://www.ebi.ac.uk/ena/data/view/PRJEB3381

When building each dataset, we concatenate its sequences using a separator that is not in the alphabet, replacing runs of undetermined characters with a single occurrence of the separator. We build the dataset of repetitive proteins as follows. We sort all bacterial protein clusters in $\mathrm{NCBI}^{4}$ (which are sets of protein sequences with high pairwise alignment scores) by decreasing number of sequences in a cluster. For every such cluster, we concatenate all sequences in it, in order, until we reach approximately 70 million characters.

In all datasets we select contexts using the four-thresholds criterion, with the parameters $\tau_{1}=10^{-4}, \tau_{2}=10^{-3}, \tau_{3}=0.952, \tau_{4}=1.05$ used for proteins in [5] (similar values are also used in $[23,3,22,6])$. Using different criteria does not fundamentally change our results.

\section{Index construction}

We sketch some techniques that we observed are effective for building VOMMs in practice.

\subsection{Balanced parentheses}

We build the balanced parentheses representation of the full topology of $\overline{\mathrm{ST}}$ (without pruning) by iterating over all left-maximal substrings of $T$ with BWT, as described in $[7$, Section 4.1]. Specifically, we compute the lexicographic range $\left[i_{W} . . j_{W}\right]$ in BWT of every leftmaximal substring $W$. Let open[1..|T|] (respectively, close[1..|T|]) be an array that stores the number of substrings $W$ such that $i_{W}=i$ (respectively, $j_{W}=j$ ). Given such arrays, one could build the balanced parentheses representation of ST by iteratively printing open $[i]$ open parentheses followed by close $[i]$ closed parentheses, for all values of $i$. To build open and close we could just increment open $\left[i_{W}\right]$ and close $\left[j_{W}\right]$ for each traversed $W$. Such vectors take $|T| \log |T|$ bits of space, so it is desirable to make them smaller. The first idea to reduce their space in practice is based on the fact that $\min ($ open $[i]$, close $[i])<2$ for every $i$, since otherwise $\overline{\mathrm{ST}}$ would have a unary path. We can thus encode both open $[i]$ and close $[i]$ in a single array of counters openClose[1..|T|], using e.g. the following encoding: a zero at position $i$ means that there is no open and no closed parenthesis at $i$; a one means that there is just one open parenthesis; a two that there is just one closed parenthesis; a three that there are one open and one closed parenthesis; an even number $n \geq 4$ means that there are $n / 2$ open parentheses and one closed parenthesis; an odd number $n \geq 5$ means that there

\footnotetext{
${ }^{4}$ https://www.ncbi.nlm.nih.gov/proteinclusters
} 
are $(n-1) / 2$ closed parentheses and one open parenthesis. Note also that in practice most counters are small, thus we use a two-level scheme, in which an array stores values of length at most $b$ bits, and a hash table stores all other values ${ }^{5}$. We can store approximately $2^{b-1}$ parentheses per position of the array: if we need more, we mark the position as saturated and we add a new counter to the hash table, where keys are BWT positions and values are 64-bit integers. We could use a similar algorithm to build the balanced parentheses representation of the full topology of SLT (without pruning), except that now we would iterate over all right-maximal substrings of $T$ using BWT, incrementing counts in BWT using the bidirectional BWT index to synchronize the two intervals. However, since the stack of the iterator is small in practice, we just perform a preorder traversal of SLT and append open and closed parentheses, building bitvector mrSLT at the same time.

To build bitvector mrST, we traverse the topology of $\overline{\mathrm{ST}}$ in any order, we use the topology to get the interval $[i . . j]$ of each node in BWT, and we check the number of ones in the interval $[i+1 . . j]$ inside an additional bitvector $\operatorname{diff}[1 . .|T|]$ such that $\operatorname{diff}[i]=1$ iff $\overline{\mathrm{BWT}}[i] \neq \overline{\mathrm{BWT}}[i-1][11]$.

We build the data structures in Lemma 1 of the paper as follows. We build the balanced parentheses representation of the topologies of $\overline{\mathrm{ST}}$ and SLT like in the non-pruned case by traversing SLT using the birectional BWT index, but keeping only nodes that are maximal repeats or left-extensions of maximal repeats. We also mark the required bits in leafToMaxrep during the traversal.

\subsection{Complexity and comparison to the competitors}

We can measure most quantities used in Section 2.3 of the paper to decide if a node of $\overline{\mathrm{ST}}$ is a context, within the same time and space budget as traversing the tree using $\overline{\mathrm{BWT}}$. Thus, for most variants, construction takes $O(|T| \log \sigma)$ time. In general, construction takes $O(v|T| \log \sigma)$ time, where $v$ is the time to decide whether a node of $\overline{\mathrm{ST}}$ is associated with a context.

Computing the contexts for a different setting of the context selection thresholds amounts just to a traversal of SLT, thus re-training a VOMM that was already built is significantly faster than training one from scratch. For the same reason, storing multiple VOMMs trained on the same dataset with different settings takes significantly less space than the sum of the individual VOMMs.

On an Intel Xeon E7-4830v3 at 2.10 GHz with one terabyte of RAM, our sequential construction algorithm takes approximately ten hours to index the human genome, and between three and four days to index all bacteria, all proteins, the metagenome, and the read set. Trie-based data structures are from 5 to more than 290 times slower to build than our index in practice, depending on the number of contexts (Figure 1). Building the index of [24] is approximately 3 times faster than building our non-pruned index, and 5 times

\footnotetext{
${ }^{5}$ We observe that setting $b=8$ achieves a good balance between the size of the array and the number of elements in the hash table.
} 
faster than building our pruned index, except for aggressive depth pruning (Figure 2), but our construction algorithm uses approximately half the space of the competitor. Once our index is built, creating one with a different context selection criterion is faster than building the competitor from scratch, and it takes half the space required for building our index from scratch. Building the index in [32] takes between 5 and 9 bytes per character, which is comparable to our construction, and between 1.1 and 3.2 microseconds per character, which is faster than or comparable to our non-pruned index.

Note that, once our indexes are built, the user can also discard the training data and use the index itself to reconstruct $T$ if needed. Note also that all construction algorithms could be parallelized, by performing parallel traversals of ST and of SLT (see e.g. [1]).

\section{Scoring time}

We implement just basic versions of each scoring criterion, paying attention only at minimizing the number of conversions between node identifier in a topology and corresponding BWT interval. Clearly the query $S$ does not need to be kept in memory but can be streamed from disk.

\subsection{Comparison to the competitors}

As mentioned, our non-pruned index is between 60 and 35 times slower than the trie-based index in [4] (Figure 3). Contrary to the competitors, however, scoring time decreases when the number of contexts increases: this is likely due to the fact that, when only few contexts are selected, most matching statistics strings need to issue a lowest marked ancestor query to jump to their context, whereas when many contexts are selected, it is more likely for a matching statistics string to already be a context, and thus to avoid a lowest marked ancestor query.

Recall that the implementation in [24] cannot index strings longer than approximately 80 million characters. Thus, we compare the speed of scoring with our index and with the competitor, by using the following small datasets as indexes: (1) a random subset of all NCBI proteins, of length 80 million; (2) the set of repetitive proteins described above; (3) a random subset of bacterial genomes, of length 80 million; (4) a prefix of the $H$. influenzae file, of length 80 million. We use such datasets to cover the cases of repetitive and nonrepetitive indexes. We use as queries ten random subsets of all NCBI proteins, and ten random subsets of all bacterial genomes, of length 500 million each. Recall also that the competitor does not allow one to use contexts to score a query; instead, it just computes the ratio between the frequency of $S[i-\mathrm{MS}[i]+1 . . i]$ and the frequency of $S[i-\mathrm{MS}[i]+1 . . i-1]$ for each $i$. We implement such criterion using an even smaller data structure, since we don't need to measure string depths. Results are in Figure 5. We don't compare scoring time to [32], since the latter supports just one scoring function which is significantly different from the ones we consider. 


\subsection{Recursive scoring}

It is interesting to compare recursive and non-recursive scoring time when the number of contexts increases. As before, we decrease threshold $\tau_{1}$ in the four-thresholds selection criterion of Section 2.2.2 in the paper. When $\tau_{1}$ is large, recursive and non-recursive scores take similar time, since we are likely selecting very short contexts, which are probably followed by most characters of the alphabet, and thus recursion stops soon (see Figure 5). As expected, when $\tau_{1}$ decreases, the recursive score becomes slower, since more substrings are marked as contexts, thus the matching statistics string at each position of the query tends to match a longer context, which is more likely to emit just few characters, and thus recursion is more likely to continue upwards in ST. We observe the recursive score being up to 3.5 times slower than the non-recursive score, and the gap widens as $\tau_{1}$ decreases.

\section{3 $\quad$ Speeding up scoring}

Slower query times could be mitigated in practice by applying optimizations from matching statistics (like those in [9], some of which are implicitly enabled by our pruned topologies already), by precomputing counts that are too expensive to evaluate at query time (as done e.g. in $[21,30,31,32])$, and by taking advantage of the large number of cores that are standard in current servers. Scoring is indeed embarrassingly parallel in most applications, where the dataset to be queried is a large number of short strings, like sequencing reads or proteins. If $S$ is one long string, one can still split it in uniform blocks and parallelize matching statistics.

\section{Explicit storage of string depths}

Figure 6 shows the advantages of a naive explicit encoding of the lengths of maximal repeats. Alternatively, we could use a multilevel scheme like directly addressable codes [12], which have already been used e.g. by [32] for storing precomputed counts. Specifically, a first array could contain one byte for every maximal repeat in preorder, and the first bit in such byte could mark whether the length of the maximal repeat is longer than $2^{7}$. A second array stores again one byte for every maximal repeat of length greater than $2^{7}$, and uses again the first bit to mark whether the length is greater than $2^{14}$. A third array contains the remaining $\log d-14$ bits that encode the length of every maximal repeat of length greater than $2^{14}$, where $d$ is the maximum length of a maximal repeat. To move from one array to the other, we could store partial rank information every few bytes.

Another alternative could be to simply store the difference between the string depths of maximal repeats that are consecutive in preorder, using two bitvectors add and subtract, each containing exactly a number of ones equal to the number of maximal repeats: if the difference between the string depths of node $i$ and $i-1$ in preorder is equal to $d$, we append $d$ zeros followed by a one to add, and if it is equal to $-d$ we append $d$ zeros followed by a 
one to subtract.

Note that storing depths explicitly might reduce query time, since fewer operations are needed to access the length of a maximal repeat. In practice we observe negligible speedups in all datasets, even with recursive scores, suggesting that string depth retrieval is not a bottleneck of score computation.

\section{$9 \quad$ Background on string indexes}

We briefly summarize here some background notions on data structures that are required for understanding the paper.

Let $T \in[1 . . \sigma]^{n-1} \#$ be a string. A rank query $\operatorname{rank}(T, a, i)$ returns the number of occurrences of character $a$ in $T$ up to position $i$, inclusive. A select query $\operatorname{select}(T, a, i)$ returns the position of the $i$-th occurrence of $a$ in T. See e.g. [17]. The suffix array $\mathrm{SA}_{T}[1 . .|T|]$ of $T$ is the vector of indices such that $T\left[\mathrm{SA}_{T}[i] . .|T|\right]$ is the $i$-th smallest suffix of $T$ in lexicographic order. The Burrows-Wheeler transform of $T$ is the string $\mathrm{BWT}_{T}[1 . .|T|]$ satisfying $\mathrm{BWT}_{T}[i]=T\left[\mathrm{SA}_{T}[i]-1\right]$ if $\mathrm{SA}_{T}[i]>1$, and $\mathrm{BWT}_{T}[i]=\#$ otherwise [13]. While $\mathrm{SA}_{T}$ takes $|T| \log |T|$ bits, $\mathrm{BWT}_{T}$ takes $|T| \log \sigma$ bits, i.e. the same number of bits needed to store $T$. A substring $W$ of $T$ can be represented as a lexicographic interval $[i . . j]$ of suffixes in both $\mathrm{SA}_{T}$ and $\mathrm{BWT}_{T}$. Array $C[0 . . \sigma]$ stores in $C[a]$ the number of occurrences in $T$ of all characters strictly smaller than $a$, i.e. the sum of the frequency of all characters in set $\{\#, 1, \ldots, a-1\}$. Clearly $C[0]=0$, and $C[a]+1$ is the position in $\mathrm{SA}_{T}$ of the first suffix of $T$ that starts with character $a$. The wavelet tree is a data structure that represents a string $T$ in $|T| \log \sigma(1+o(1))$ bits and supports rank, select, and access operations on its characters in $O(\log \sigma)$ time (see e.g. [19]). The combination of BWT with rank support and $C$ array is known as FM-index, and it enables a backward step, i.e. moving from the interval $[i . . j]$ of a substring $W$ of $T$, to the interval $\left[i^{\prime} . . j^{\prime}\right]$ of its left extension $a W$, where $a$ is a character [16].

We also require familiarity with the notion and usages of the suffix tree $\mathrm{ST}_{T}=(V, E)$ of $T \#$ [33]: see e.g. [20] for an overview. We denote by $\overline{\mathrm{ST}}_{T}$ the suffix tree of $\bar{T} \#$, and we denote by $\ell(v)$ the string label of a node $v \in V$, i.e. the string obtained by concatenating the labels of all edges in the path from the root of the tree to $v$. Here we just recall that a substring $W$ of $T$ is right-maximal (respectively, left-maximal) iff $W=\ell(v)$ for some internal node $v$ of $\mathbf{S T}_{T}$ (respectively, for some internal node $v$ of $\overline{\mathbf{S T}}_{T}$ ), and that a node $v \in V$ with $\ell(v)=a W$ for some character $a \in[0 . . \sigma]$ points to a node $w \in V$ with $\ell(w)=W$ by a suffix link labeled by $a$. Suffix links and internal nodes of $\mathbf{S T}_{T}$ form a trie, called the suffix-link tree of $T$ and denoted by $\mathrm{SLT}_{T}$. Inverting the direction of all suffix links yields the so-called explicit Weiner links. Given an internal node $v$ and a symbol $a \in[0 . . \sigma]$, it might happen that string $a \ell(v)$ occurs in $T$, but is not right-maximal, i.e. it is not the label of any internal node of ST: all such left extensions of internal nodes that end in the middle of an edge are called implicit Weiner links. An internal node $v$ of 
ST can have more than one outgoing Weiner link, and all such Weiner links have distinct labels: in this case, $\ell(v)$ is a maximal repeat. We call $S_{L}^{*} T_{T}^{*}$ a version of $\operatorname{SLT}_{T}$ augmented with implicit Weiner links and with nodes corresponding to their destinations. A maximal repeat $W$ of $T$ is called leftmost if it is not the proper suffix of any other maximal repeat of $T$. Since taking the suffix of a string preserves right-maximality, the set of all maximal repeats coincides with the set of all ancestors in $\overline{\mathrm{ST}}$ of leftmost maximal repeats. It is easy to see that there is a bijection between the set of branching nodes of SLT* and the nodes of $\overline{\mathrm{ST}}$ that correspond to maximal repeats, in which the leaves of SLT are mapped to the nodes of $\overline{\mathrm{ST}}$ that correspond to leftmost maximal repeats. The suffix-link tree (a trie) is thus a subdivision of the subgraph of $\overline{\mathrm{ST}}$ induced by maximal repeats (a compact tree). See Section 2.1 in [8] for a more thorough explanation.

The topology of an ordered tree with $n$ nodes can be represented using $2 n+o(n)$ bits, as a sequence of $2 n$ balanced parentheses built by opening a parenthesis, by recurring on every child of the current node in order, and by closing a parenthesis [25]. Let id $(v)$ be the rank of a node $v$ in the preorder traversal of the tree. Given the balanced parentheses representation of the tree encoded in $2 n+o(n)$ bits, one can build a data structure that takes $2 n+o(n)$ bits, and that supports the following operations in constant time [26, 28, 27]:

- $\operatorname{child}(\operatorname{id}(v), i)$ : returns $\operatorname{id}(w)$, where $w$ is the $i$ th child of node $v(i \geq 1)$, or $\emptyset$ if $v$ has less than $i$ children;

- $\operatorname{parent}(\operatorname{id}(v))$ : returns $\operatorname{id}(u)$, where $u$ is the parent of $v$, or $\emptyset$ if $v$ is the root of $T$;

- $\operatorname{lca}(\operatorname{id}(v), \operatorname{id}(w))$ : returns $\operatorname{id}(u)$, where $u$ is the lowest common ancestor of nodes $v$ and $w$;

- leftmostLeaf $(\operatorname{id}(v))$, rightmostLeaf $(\operatorname{id}(v))$ : returns one plus the number of leaves that, in the preorder traversal of $T$, are visited before the first (respectively, the last) leaf that belongs to the subtree of $T$ rooted at $v$;

- selectLeaf $(i)$ : returns $\operatorname{id}(v)$, where $v$ is the $i$-th leaf visited in the preorder traversal of $T$;

- $\operatorname{depth}(\operatorname{id}(v))$, height $(\operatorname{id}(v))$ : returns the distance of $v$ from the root or from its deepest descendant, respectively.

This data structure can be built in $O(n)$ time and in $O(n)$ bits of working space.

Note that such operations allow converting in constant time between an interval in $\mathrm{BWT}_{T}$ and the corresponding node identifier in the balanced parenthesis representation of $\mathrm{ST}_{T}$ (by selecting the corresponding leftmost and rightmost leaves and taking their lowest common ancestor), and vice versa (by counting the number of leaves under a node). 


\section{References}

[1] Jarno Alanko, Fabio Cunial, Djamal Belazzougui, and Veli Mäkinen. A framework for space-efficient read clustering in metagenomic samples. BMC Bioinformatics, 18(3):59, 2017 .

[2] Uwe Baier, Timo Beller, and Enno Ohlebusch. Space-efficient parallel construction of succinct representations of suffix tree topologies. Journal of Experimental Algorithmics (JEA), 22:1-1, 2017.

[3] Ron Begleiter, Ran El-Yaniv, and Golan Yona. On prediction using variable order Markov models. Journal of Artificial Intelligence Research, 22:385-421, 2004.

[4] Gill Bejerano. Algorithms for variable length Markov chain modeling. Bioinformatics, 20(5):788-789, 2004.

[5] Gill Bejerano and Golan Yona. Modeling protein families using probabilistic suffix trees. In Proceedings of the third Annual International Conference on Computational Molecular Biology, pages 15-24. ACM, 1999.

[6] Gill Bejerano and Golan Yona. Variations on probabilistic suffix trees: statistical modeling and prediction of protein families. Bioinformatics, 17(1):23-43, 2001.

[7] Djamal Belazzougui. Linear time construction of compressed text indices in compact space. In Symposium on Theory of Computing, STOC 2014, New York, NY, USA, May 31 - June 03, 2014, pages 148-193. ACM, 2014.

[8] Djamal Belazzougui and Fabio Cunial. A framework for space-efficient string kernels. Algorithmica, pages 1-27, 2017.

[9] Djamal Belazzougui, Fabio Cunial, and Olgert Denas. Fast matching statistics in small space. In Proceedings of the 17th International Symposium on Experimental Algorithms (SEA 2018), 2018.

[10] Djamal Belazzougui, Fabio Cunial, Travis Gagie, Nicola Prezza, and Mathieu Raffinot. Composite repetition-aware data structures. In Annual Symposium on Combinatorial Pattern Matching, pages 26-39. Springer, 2015.

[11] Djamal Belazzougui, Fabio Cunial, Juha Kärkkäinen, and Veli Mäkinen. Versatile succinct representations of the bidirectional Burrows-Wheeler transform. In AlgorithmsESA 2013, pages 133-144. Springer, 2013.

[12] Nieves R Brisaboa, Susana Ladra, and Gonzalo Navarro. Dacs: Bringing direct access to variable-length codes. Information Processing $\&$ Management, 49(1):392-404, 2013. 
[13] M. Burrows and D. Wheeler. A block sorting lossless data compression algorithm. Technical Report 124, Digital Equipment Corporation, 1994.

[14] Imre Csiszár and Paul C Shields. The consistency of the BIC Markov order estimator. Annals of Statistics, pages 1601-1619, 2000.

[15] Meir Feder, Neri Merhav, and Michael Gutman. Universal prediction of individual sequences. IEEE Transactions on Information Theory, 38(4):1258-1270, 1992.

[16] P. Ferragina and G. Manzini. Indexing compressed texts. Journal of the ACM, $52(4): 552-581,2005$.

[17] Travis Gagie. Rank and select operations on sequences. In Encyclopedia of Algorithms, pages 1776-1780. Springer, 2016.

[18] Simon Gog, Timo Beller, Alistair Moffat, and Matthias Petri. From theory to practice: plug and play with succinct data structures. In 13th International Symposium on Experimental Algorithms (SEA 2014), pages 326-337, 2014.

[19] Roberto Grossi. Wavelet trees. In Encyclopedia of Algorithms. Springer US, Boston, MA, 2008.

[20] Dan Gusfield. Algorithms on strings, trees and sequences: computer science and computational biology. Cambridge University Press, 1997.

[21] Christopher Kermorvant and Pierre Dupont. Mélanges de chaînes de Markov lissées pour la détection de domaines dans les protéines. In Proceedings des Journées Ouvertes Biologie Informatique Mathématique, 2002.

[22] Christine Largeron-Leténo. Prediction suffix trees for supervised classification of sequences. Pattern Recognition Letters, 24(16):3153-3164, 2003.

[23] Florencia G Leonardi. A generalization of the PST algorithm: modeling the sparse nature of protein sequences. Bioinformatics, 22(11):1302-1307, 2006.

[24] Jie Lin, Donald Adjeroh, and Bing-Hua Jiang. Probabilistic suffix array: efficient modeling and prediction of protein families. Bioinformatics, 28(10):1314-1323, 2012.

[25] J Ian Munro and Venkatesh Raman. Succinct representation of balanced parentheses and static trees. SIAM Journal on Computing, 31(3):762-776, 2001.

[26] Gonzalo Navarro. Compact data structures: A practical approach. Cambridge University Press, 2016.

[27] Gonzalo Navarro and Kunihiko Sadakane. Fully functional static and dynamic succinct trees. ACM Transactions on Algorithms, 10(3):16:1-16:39, 2014. 
[28] K. Sadakane and G. Navarro. Fully-functional succinct trees. In Proc. ACM-SIAM Symposium on Discrete Algorithms (SODA 2010), pages 134-149, Austin, Texas, USA, 2010. ACM-SIAM.

[29] Steven L Salzberg, Arthur L Delcher, Simon Kasif, and Owen White. Microbial gene identification using interpolated Markov models. Nucleic Acids Research, 26(2):544$548,1998$.

[30] Ehsan Shareghi, Trevor Cohn, and Gholamreza Haffari. Richer interpolative smoothing based on modified Kneser-Ney language modeling. In Proceedings of the 2016 Conference on Empirical Methods in Natural Language Processing, pages 944-949, 2016.

[31] Ehsan Shareghi, Matthias Petri, Gholamreza Haffari, and Trevor Cohn. Compact, efficient and unlimited capacity: language modeling with compressed suffix trees. In Proceedings of the 2015 Conference on Empirical Methods in Natural Language Processing, pages 2409-2418, 2015.

[32] Ehsan Shareghi, Matthias Petri, Gholamreza Haffari, and Trevor Cohn. Fast, small and exact: infinite-order language modelling with compressed suffix trees. Transactions of the Association for Computational Linguistics, 4:477-490, 2016.

[33] P. Weiner. Linear pattern matching algorithm. In Proc. 14th Annual IEEE Symposium on Switching and Automata Theory, pages 1-11, 1973. 
bioRxiv preprint doi: https://doi.org/10.1101/443101; this version posted October 14,2018 . The copyright holder for this preprint (which was not certified by peer review) is the author/funder, who has granted bioRxiv a license to display the preprint in perpetuity. It is made available under aCC-BY-ND 4.0 International license.

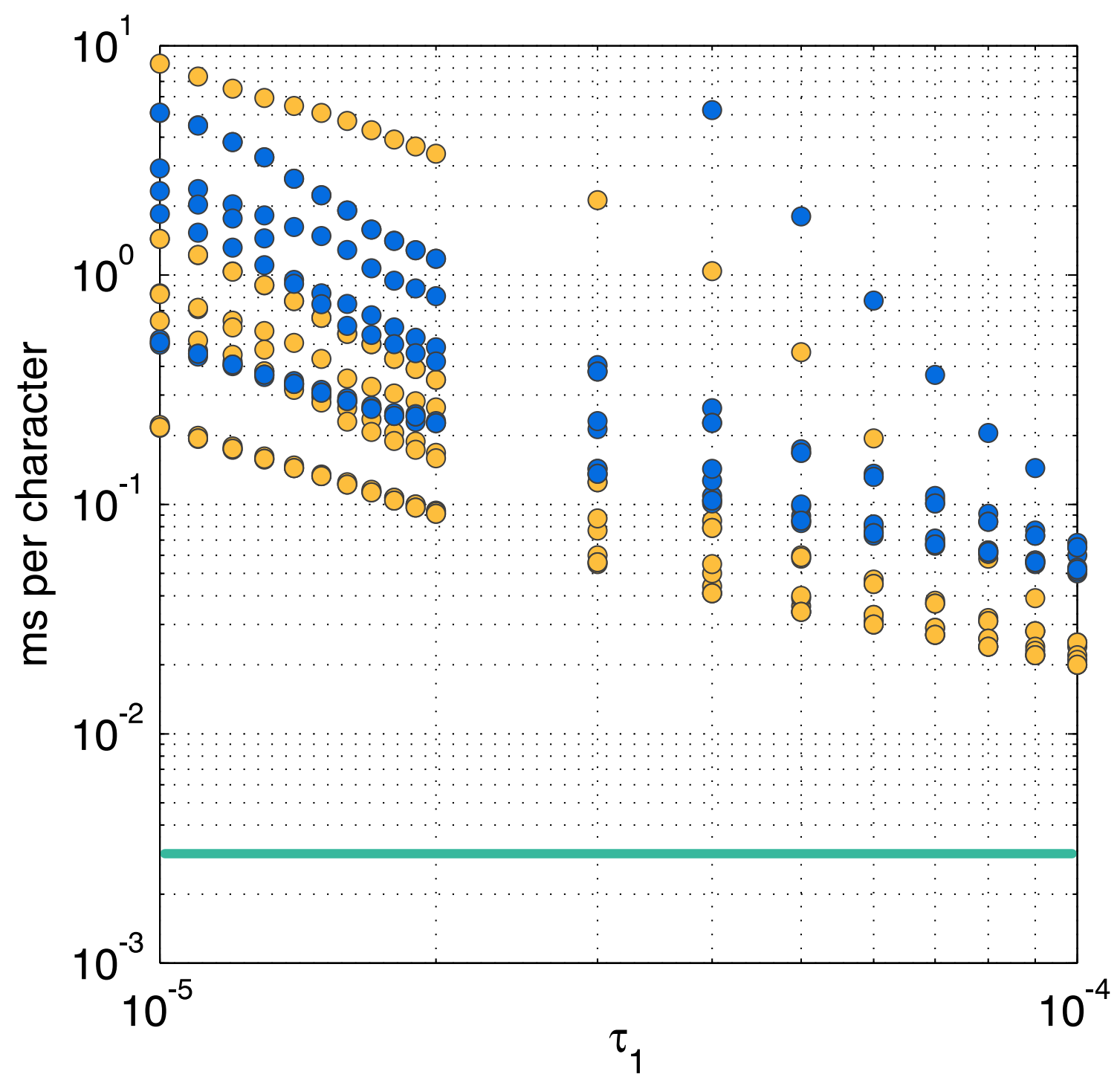

Figure 1: Time for building trie representations of VOMMs as a function of $\tau_{1}$ in the fourthresholds context selection criterion. The figure follows the conventions of Figure 1 in the paper. 
bioRxiv preprint doi: https://doi.org/10.1101/443101; this version posted October 14,2018 . The copyright holder for this preprint (which was not certified by peer review) is the author/funder, who has granted bioRxiv a license to display the preprint in perpetuity. It is made available under aCC-BY-ND 4.0 International license.
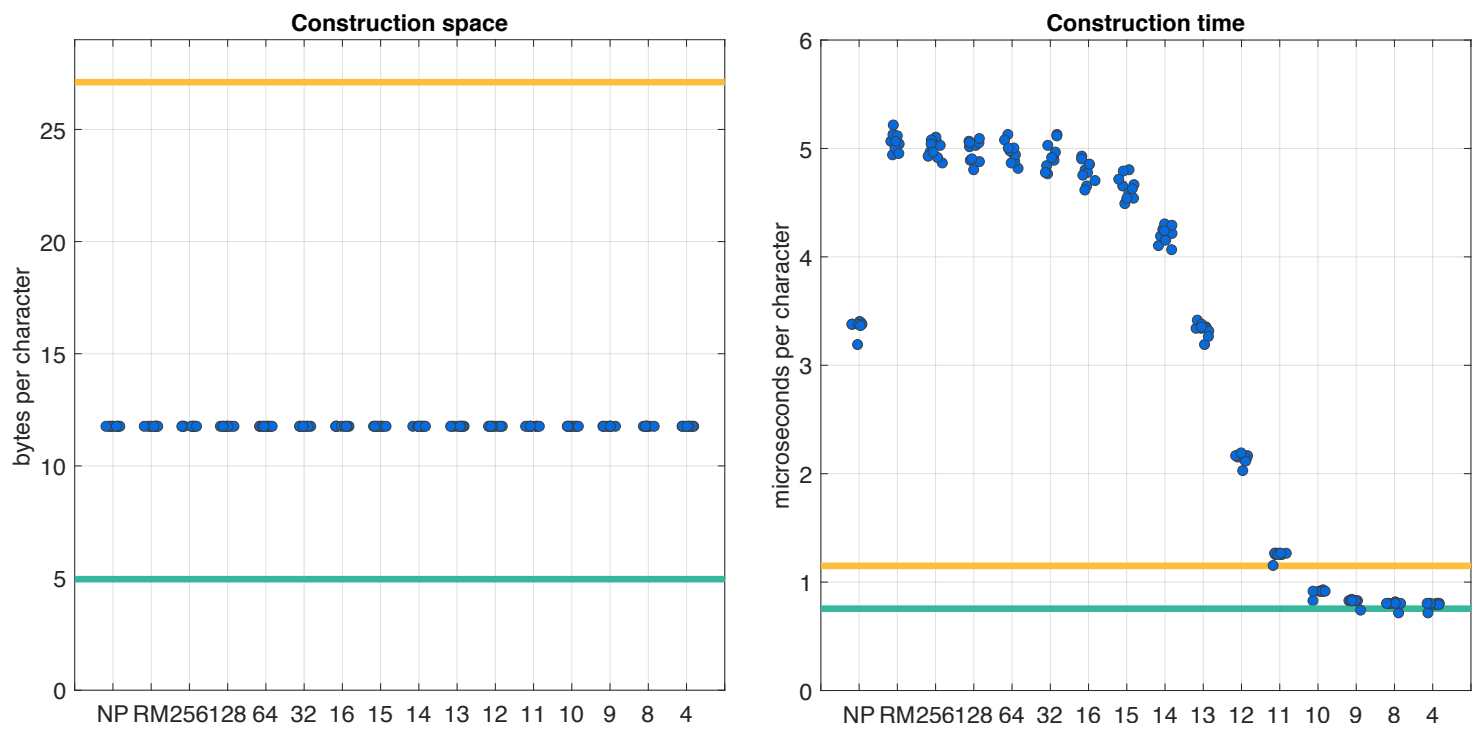

Figure 2: Peak space and total running time of our construction programs (blue circles), compared to [24]. Orange lines: average of the competitor. Green lines: average of our reconstruction program. Dataset: random substrings of the concatenation of all bacterial genomes. 


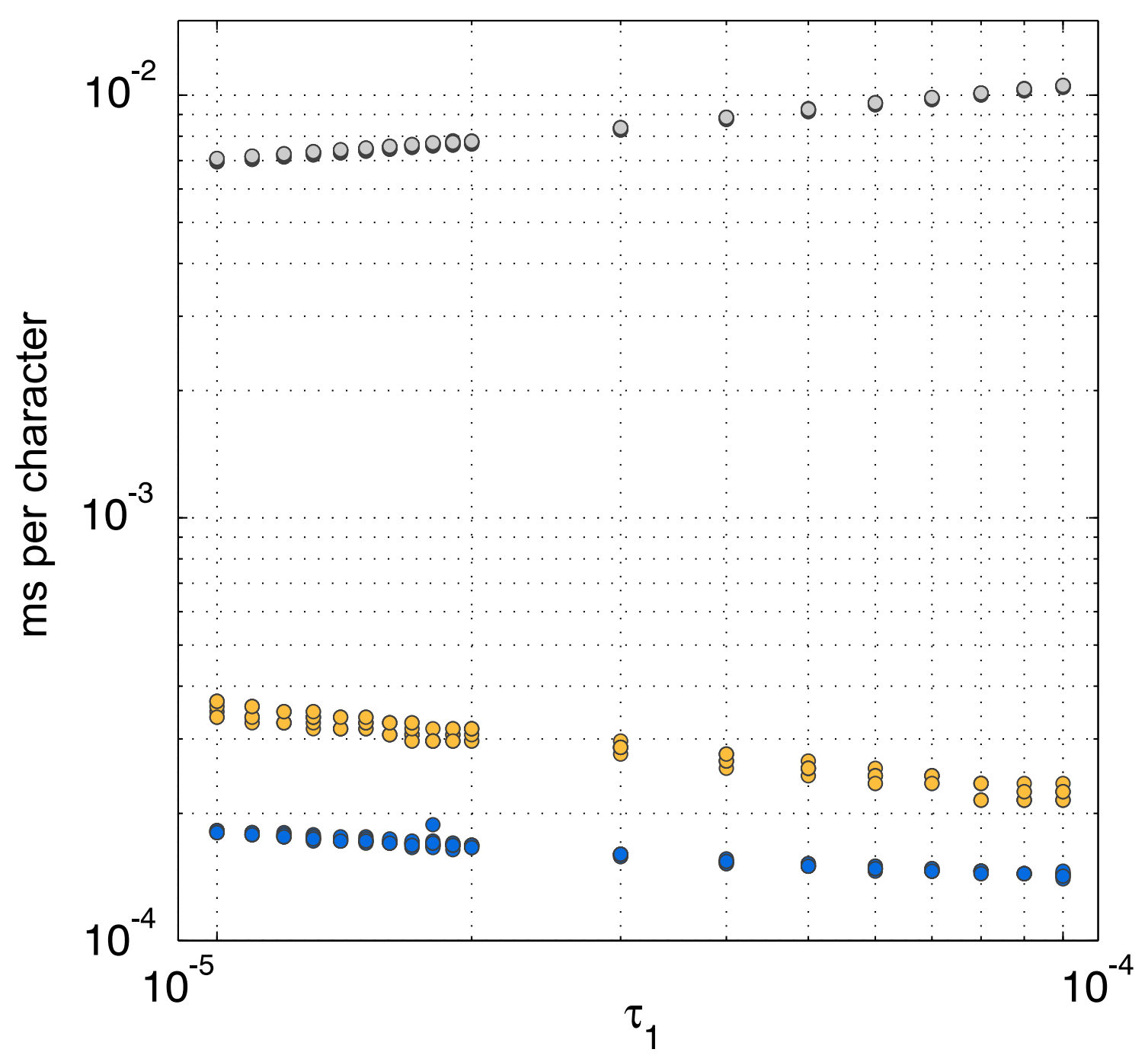

Figure 3: Scoring time: comparison between our index (grey) and the implementations in [4] (blue) and [23] (orange). Query datasets: ten nonrepetitive concatenations of proteins of length 500 million each. Index datasets: the same as in Figure 1 of the paper. 


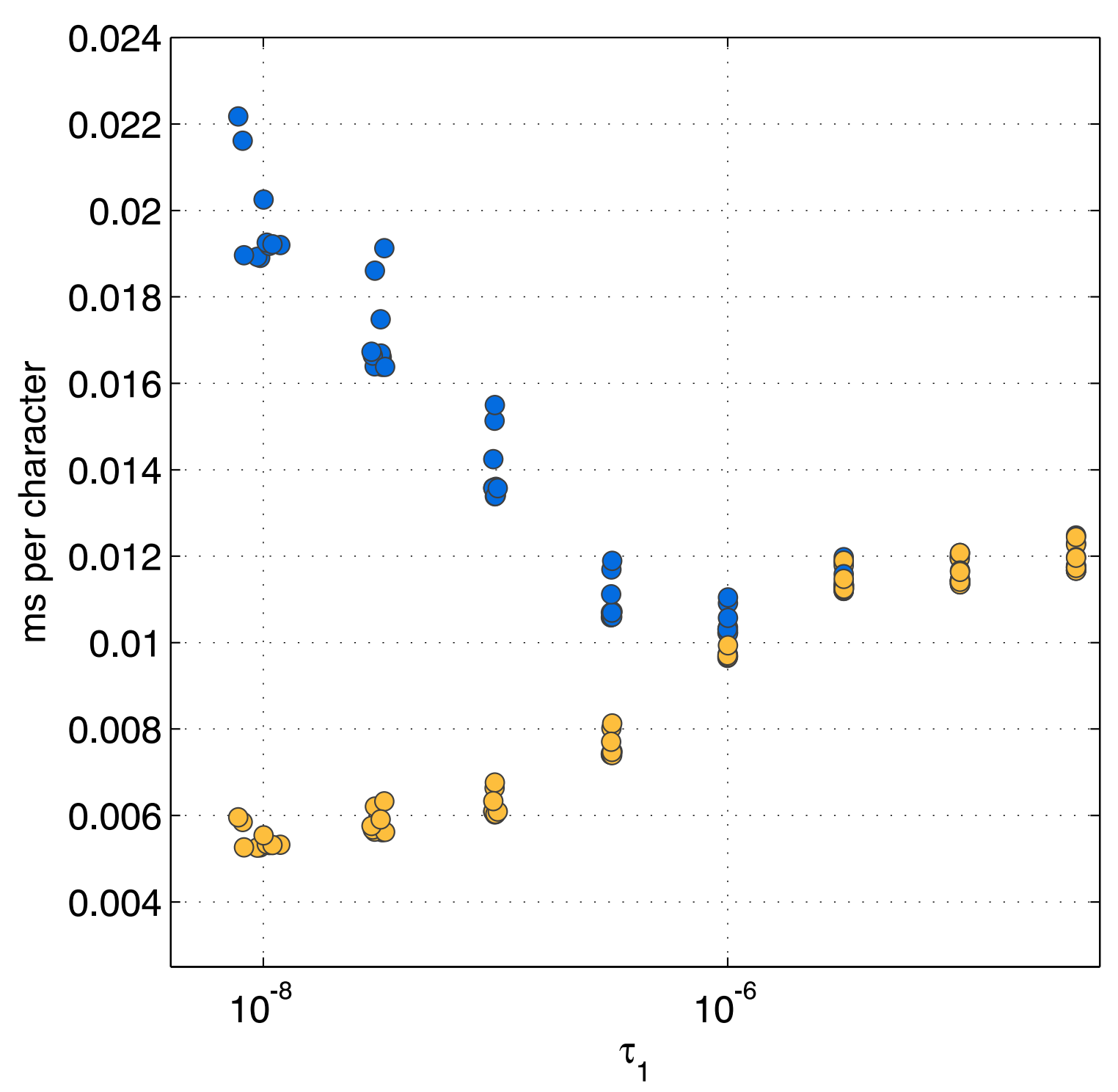

Figure 4: Recursive (blue circles) and non-recursive (orange circles) scoring in our implementation, as a function of $\tau_{1}$ in Section 2.2.2. of the paper (horizontal axis, logarithmic scale). Queries: ten non-repetitive concatenations of proteins of length 500 million. Index: one non-repetitive concatenation of proteins of length 80 million. Experiments with repetitive proteins yield similar results. 

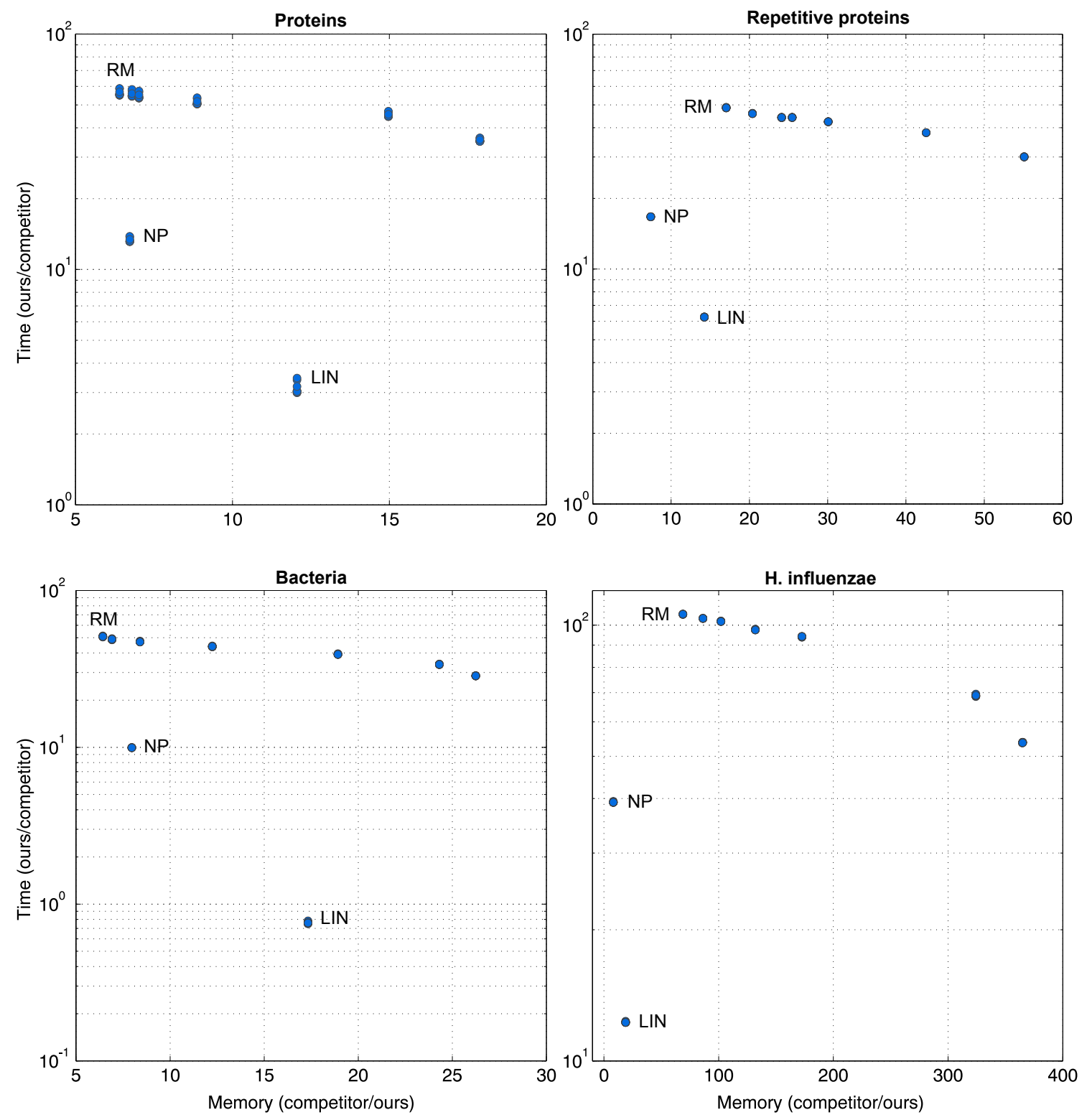

Figure 5: Scoring time: comparison between our index and the implementation in [24]. LIN: score function defined by the competitor. NP: context-based scoring, no pruning. RM: context-based scoring, run-length encoding and maximal repeat pruning. Unlabeled points correspond to RM with decreasing maximum context length (from left to right). Each configuration is tested with ten random substrings, whose measurements are highly overlapping. Memory improvements do not reflect those in Figure 3 of the paper, since the datasets used here are subsets of length at most 80 million of the full datasets. 


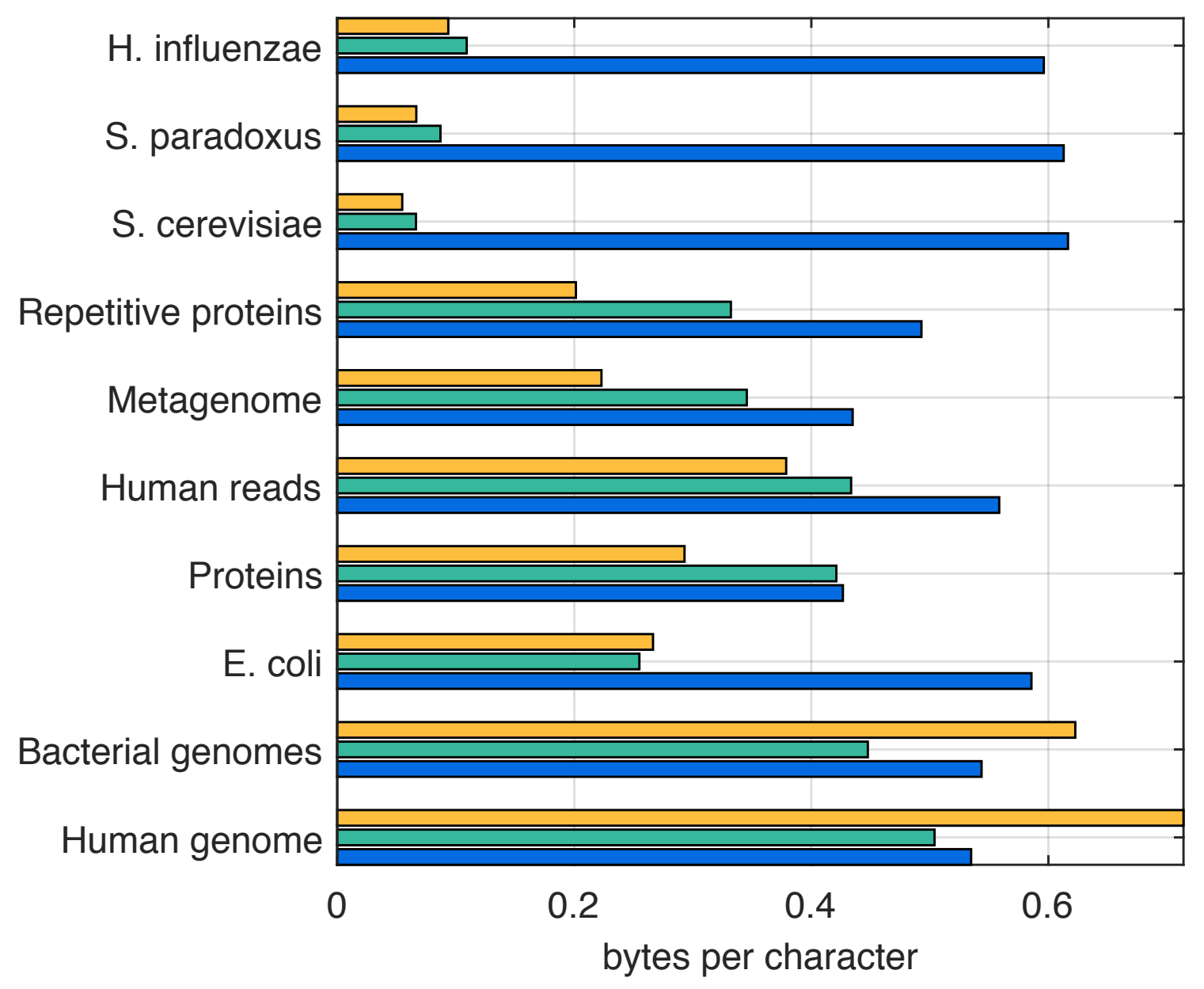

Figure 6: Size of the explicit storage of string depths (orange), compared to the size of the uncompressed (blue) and compressed (green) SLT. Topologies are not pruned. 